\title{
Caractéristiques hydro-géomorphologiques des microhabitats d'Unio crassus (Ardenne, Belgique)
}

Hydro-geomorphological characteristics of Unio crassus microhabitats (Ardenne, Belgium)

Quintia Vaessen, Geoffrey Houbrechts, Alexandre Peeters et Jean Van Campenhout

\section{(2) OpenEdition}

\section{Journals}

Édition électronique

URL : https://journals.openedition.org/geomorphologie/15113

DOI : $10.4000 /$ geomorphologie. 15113

ISSN : 1957-777X

Éditeur

Groupe français de géomorphologie

Édition imprimée

Date de publication : 30 avril 2021

Pagination : $3-18$

ISBN : 978-2-913282-90-2

ISSN : 1266-5304

Ce document vous est offert par Université de Liège

\section{LIÈGE}

université

Référence électronique

Quintia Vaessen, Geoffrey Houbrechts, Alexandre Peeters et Jean Van Campenhout,

"Caractéristiques hydro-géomorphologiques des microhabitats d'Unio crassus (Ardenne, Belgique)», Géomorphologie : relief, processus, environnement [En ligne], vol. 27 - $n^{\circ} 1$ | 2021, mis en ligne le 12 janvier 2021, consulté le 12 mai 2021. URL : http://journals.openedition.org/geomorphologie/15113 ; DOI : https://doi.org/10.4000/geomorphologie.15113 


\title{
Caractéristiques hydro-géomorphologiques des microhabitats d'Unio crassus (Ardenne, Belgique)
}

\section{Hydro-geomorphological characteristics of Unio crassus microhabitats (Ardenne, Belgium)}

\author{
Quintia Vaessen*, Geoffrey Houbrechts, Alexandre Peeters, Jean Van Campenhout
}

Laboratoire d’hydrographie et de géomorphologie fluviatile (LHGF) - Université de Liège, Département de géographie, UR Sphères, Clos Mercator 3, 4000 Liège, Belgique

\begin{abstract}
RÉSUMÉ
Actuellement en Europe, les populations d'Unio crassus (Bivalvia : Unionidae) déclinent rapidement. Afin de contrer cette tendance, de nombreuses actions de conservation sont mises en place. Toutefois, l'efficacité de ces actions est dépendante de notre connaissance de l'écologie de l'espèce. Cette étude se concentre sur les préférences hydromorphologiques d'U. crassus dans une rivière du sud-est de la Belgique (la Sûre). La profondeur et les vitesses de courant ont été cartographiées au niveau de 3 sites détude. Ces données ont ensuite été croisées avec la répartition des individus sur ces sites afin de mettre en relation l'utilisation et la disponibilité des microhabitats. D’autre part, un système piégeant les sédiments fins a été développé pour quantifier le colmatage du substrat dans lequel vit l'espèce. Les individus se situent majoritairement dans des zones de courants lents $(<0,4 \mathrm{~m} / \mathrm{s})$ avec des profondeurs intermédiaires $(0,4$ à $0,8 \mathrm{~m})$ pour des débits inférieurs au module. La profondeur apparait comme étant le paramètre principal gouvernant la répartition spatiale des individus à léchelle locale comparée à la vitesse de courant et la proximité à la berge. Les substrats occupés par $U$. crassus sont variés avec cependant une densité d'individus plus élevée dans des dépôts de lordre de $3 \mathrm{~mm}$ de $D_{50}$ et de $26 \mathrm{~mm}$ de $D_{99}$. L'infiltration de sédiments fins dans le lit caillouteux est relativement peu importante au niveau des sites investigués. Les résultats montrent toutefois une certaine variabilité au cours de létude. Cette étude confirme la capacité d'U. crassus à se développer dans des conditions hydromorphologiques variées, tout en soulignant l'importance des microhabitats lentiques relativement
\end{abstract} stables.

Mots-clés : moule d'eau douce, microhabitat, sédiment, préférence d'habitat, Unio crassus.

\begin{abstract}
Populations of Unio crassus (Bivalvia: Unionidae) are sharply declining in Europe. To reverse this trend, many conservation actions are being implemented. However, the effectiveness of these actions depends on our knowledge of the ecology of the species. This study focuses on the hydromorphological preferences of $\mathrm{U}$. crassus in a river in south-eastern Belgium (the Surre River). Depth and flow velocities were mapped at 3 study sites. These data were then cross-referenced with the spatial distribution of individuals at these sites in order to relate the use and availability of microhabitats. In addition, a fine sediment sampling system was developed to quantify the clogging of the substrate in which the species lives. The individuals are mostly located in areas with slow currents $(<0.4 \mathrm{~m} / \mathrm{s})$ with intermediate depths $(0.4$ to $0.8 \mathrm{~m})$ for flows below the mean annual flow. Depth appears to be the main parameter governing the spatial distribution of individuals at the local scale compared to flow velocity and proximity to the bank. The substrates occupied by U. crassus are varied, with a higher density of individuals in deposits of about $3 \mathrm{~mm}$ of $D_{50}$ and $26 \mathrm{~mm}$ of $D_{99}$. The infiltration of fine sediments into the gravel-bed layer is relatively low at the investigated sites although variability was observed between the study periods. This study confirms the ability of $\mathrm{U}$. crassus to thrive under a variety of hydromorphological conditions, while emphasizing the importance of lentic and relatively stable microhabitats.
\end{abstract}

Keywords: freshwater mussel, microhabitat, sediment, habitat suitability, Unio crassus.
${ }^{\star}$ Auteur correspondant. Tél : +32 (0)4 3665282 ; Courriels : qvaessen@uliege.be (Q. Vaessen) G.Houbrechts@uliege.be (G. Houbrechts) A.Peeters@uliege.be (A. Peeters)

Jean.VanCampenhout@uliege.be (J.Van Campenhout)

\section{Introduction}

En Europe, les écosystèmes fluviaux ont été considérablement altérés, ce qui a entrainé la disparition d'habitats de nombreuses espèces (Geist et Hawkins, 2016 ; Díaz et al., 2019). Cette altération a des conséquences sévères pour les moules d'eau douce (i.e., Bivalvia : Unionida), qui sont des animaux sédentaires à longue durée de vie (Lopes-Lima et al., 2018). La mulette épaisse (Unio crassus, Philipsson 1788), moule d'eau douce de la famille des Unionidae, a subi une régression particulièrement importante depuis les années 1970, et fait désormais face à un risque d'extinction très élevé (Lopes-Lima et al., 2014). Pourtant ces organismes sont des éléments essentiels des écosystèmes aquatiques car ils fournissent de nombreux services écosystémiques tels que la filtration de l'eau et le recyclage des nutriments (Vaughn, 2017 ; Gutiérrez et al., 2003). Les facteurs pouvant entrainer l'extinction d'une espèce sont variés et souvent étroitement reliés, et peuvent parfois ne pas être directement apparents (Bogan, 1993). Dans le cas d'U. crassus, la 
régression des populations est probablement due à des pressions anthropiques entrainant la dégradation de son habitat, la pollution des cours d'eau, ou encore un manque de poissons hôtes (LopesLima et al., 2018). Cependant, les liens de cause à effet entre le déclin et une modification environnementale précise restent difficiles à mettre en évidence (Downing et al., 2010). En outre, les mesures de conservation des zones favorables à l'espèce sont insuffisantes (Dobler, 2019), notamment en raison des lacunes scientifiques concernant son écologie (Ferreira-Rodríguez et al., 2019). Or, en Europe, les États membres sont tenus de protéger U. crassus ainsi que son habitat en vertu de la Directive 92/43/CEE du Conseil (annexes II et IV) concernant la conservation des habitats naturels ainsi que de la faune et de la flore sauvages.

La mulette épaisse vit enfouie ou demi-enfouie dans le substrat $\mathrm{du}$ lit des rivières où elle filtre les particules présentes dans l'eau pour se nourrir. L'espèce est sédentaire ; elle peut toutefois se déplacer en cas de menaces ou de modifications de son habitat (Zając et Zając, 2011). Comme chez toutes les moules d'eau douce, son cycle de vie comprend une phase de développement sur un poisson hôte nécessaire pour la métamorphose du stade larvaire (glochidie) en jeune moule. À la différence d'autres Unionida, les glochidies d'U. crassus peuvent se fixer sur plusieurs espèces hôtes potentielles (Taeubert et al., 2012). L'espèce peut être présente dans des habitats parfois assez différents en termes de substrats, de conditions hydrologiques et de paramètres physico-chimiques, ce qui complique l'identification des paramètres indispensables pour définir un habitat optimal pour l'espèce (Hochwald, 2001 ; Denic et al., 2014 ; Gates et al., 2015). Toutefois, il est indéniable qu'il existe une relation étroite entre l'environnement et la présence/absence des moules (Schwalb et al., 2013 ; Dascher et al., 2018). Manifestement, les conditions locales de microhabitats sont essentielles pour $U$. crassus et son maintien (Inoue et al., 2017). En effet, bien que la colonisation initiale par les juvéniles soit surtout dépendante de facteurs régionaux comme la dispersion par les poissons hôtes, la croissance et la reproduction des adultes sont plutôt conditionnées par des processus environnementaux locaux (Morales et al., 2006). Ces processus sont liés à des caractéristiques telles que la pente locale, la profondeur et la vitesse du courant (Zając et al., 2011, 2018). Outre ces caractéristiques hydromorphologiques, la dynamique sédimentaire revêt également une importance particulière pour les organismes vivant dans les cours d'eau, notamment en affectant la structure et la stabilité des lits fluviaux (Knighton, 1998). Ainsi, en occupant la couche supérieure des sédiments du lit, les mulettes épaisses sont directement touchées par les mouvements de charge de fond. De plus, vu la longévité d'U. crassus (10 à 30 ans), elle doit pouvoir survivre à des événements hydrologiques exceptionnels. Ces crues peuvent impliquer un remaniement important des éléments du lit, provoquer de l'érosion, et emporter les individus conduisant ainsi à leur mort (Knighton, 1998 ; Hastie et al., 2001).

L'interconnexion des causes possibles, le cycle de vie particulier d'U. crassus, les incertitudes par rapport à son écologie, ainsi que la protection insuffisante dont elle bénéficie actuellement, sont autant d'éléments intensifiant son déclin. Dans ce contexte, la définition des interactions entre l'espèce et son environnement, et l'étude de ses microhabitats constituent des impératifs afin de maximiser les chances de succès des projets de protection et/ou de réhabilitation (Geist et Hawkins, 2016 ; Inoue et al., 2017).
L'objectif de cette étude est de définir les caractéristiques hydrogéomorphologiques des microhabitats d'U. crassus dans une rivière belge (la Sûre) qui abrite des populations fonctionnelles, en : (i) analysant la répartition spatiale des individus au niveau de plusieurs tronçons, (ii) déterminant l'importance relative des variables de microhabitats mesurées dans la présence/absence de l'espèce, et (iii) examinant le transport sédimentaire et son éventuel impact sur l'espèce.

\section{Zone d'étude}

La Sûre est une rivière transfrontalière prenant sa source dans le massif ardennais (Wallonie, Belgique), et confluant avec la Moselle au Grand-duché de Luxembourg. Dans sa partie amont (HauteSûre), des populations de plusieurs milliers d'individus d'U. crassus sont présentes (Schmitz et Krippel, 2007). Cependant en septembre 2014, un déversement accidentel d'un produit phytopharmaceutique (métazachlore) dans un petit affluent belge de la Sûre (la Moyémont) a provoqué une importante mortalité dans les populations (fig. 1). Grâce aux recensements réalisés en 2011 et 2015, le Département de l'Étude du milieu naturel et agricole du Service Public de Wallonie (DEMNA - SPW) a estimé la perte des populations à $80 \%$ dans la partie belge du cours d'eau (Motte, comm. pers.).

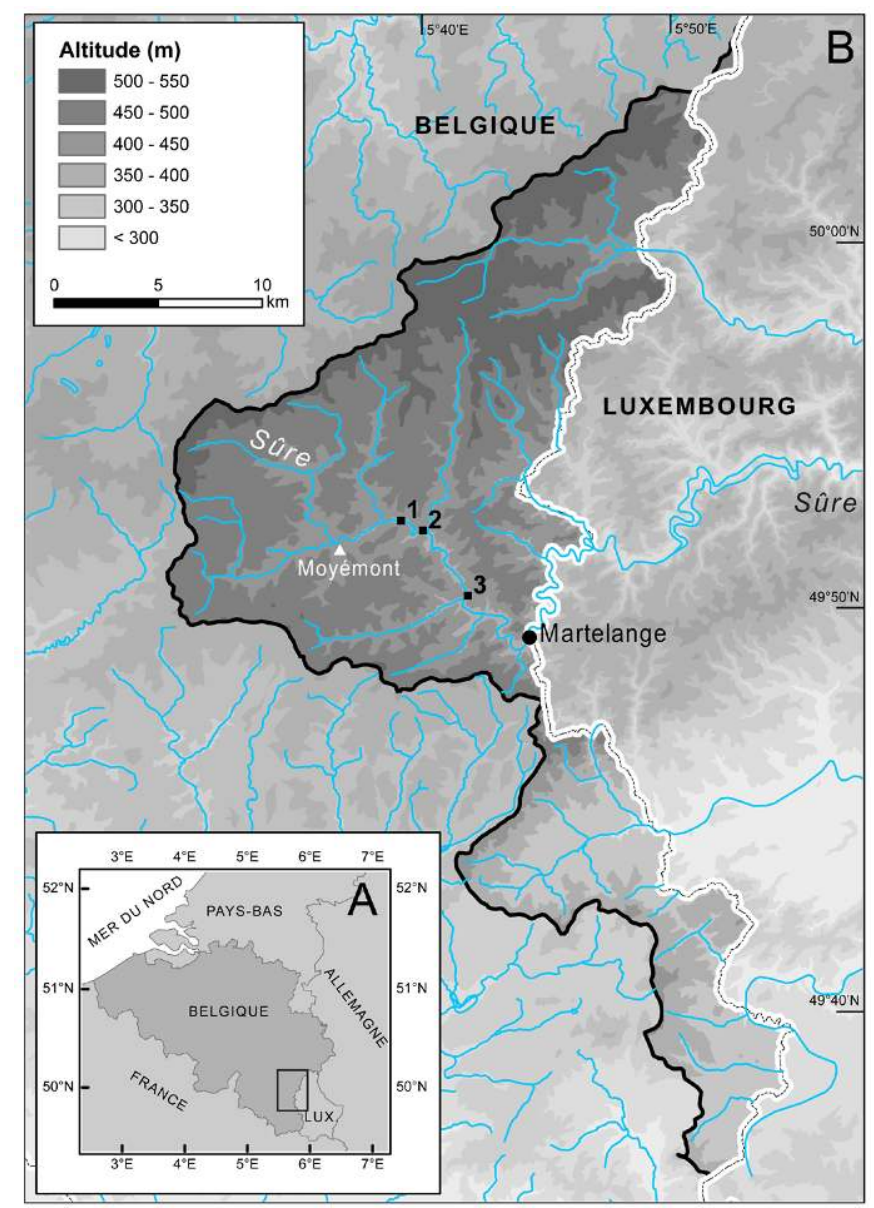

Fig. 1 - Carte de localisation.

A : Echelle régionale; B : Bassin versant de la Sûre en Belgique. 1. 2. 3. Sites d'étude Fig. 1 - Location map.

A: Regional scale; B: Sûre catchment in Belgium. 1. 2. 3. Study sites 


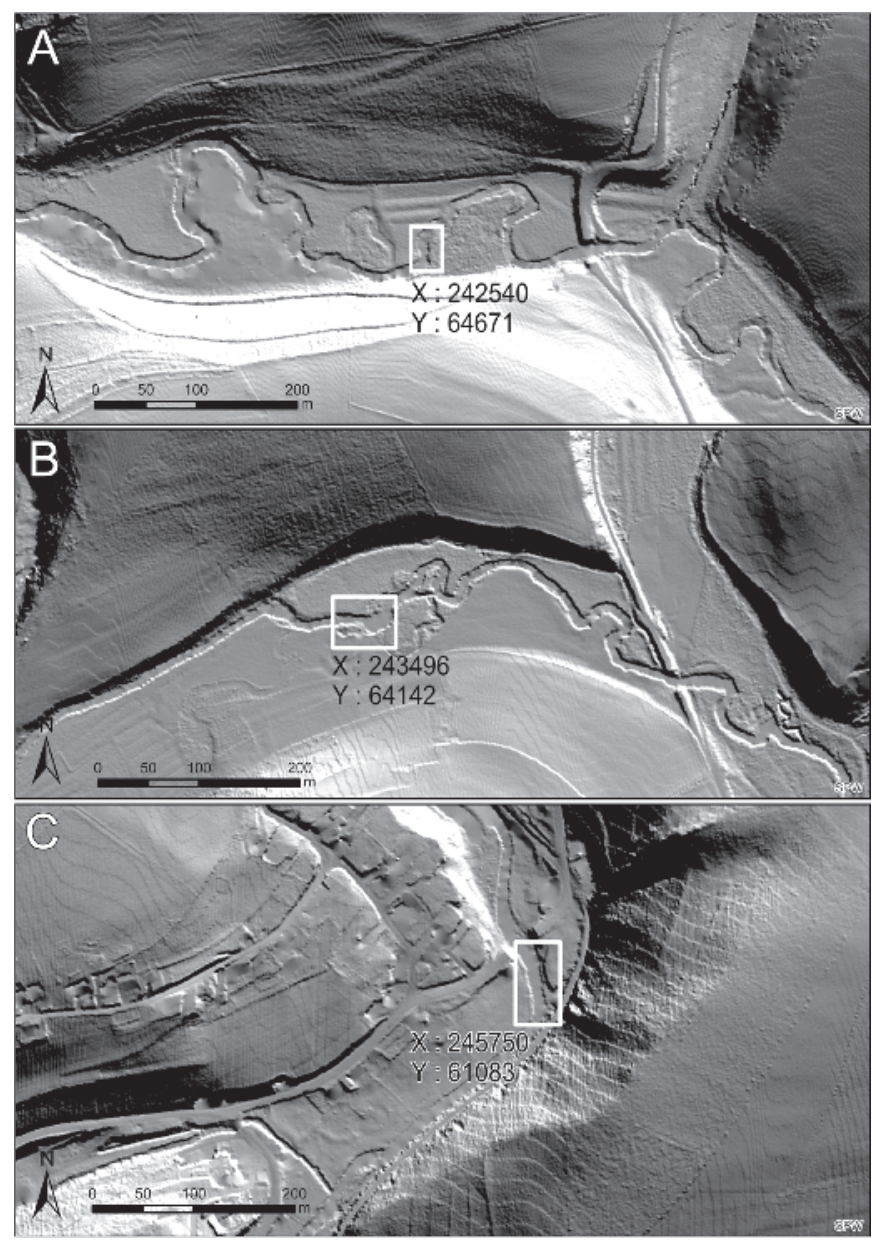

Fig. 2 - Relief des 3 secteurs avec zones d'étude. Modèle Numérique de Terrain avec interpolation hillshade obtenu via le géoportail du Service public de Wallonie (SPW).

A : Site 1 ; B : Site 2 ; C : Site 3.

Fig. 2 - Relief of the 3 study areas with sites. Digital elevation model with hillshade interpolation is from the geoportal of the Public Service of Wallonia (SPW).

A: Site $1 ; B$ : Site 2 ; C: Site 3.
La Sûre coule majoritairement sur les couches schisteuses imperméables du Dévonien inférieur. La nature imperméable de la roche mère entraîne une faible minéralisation de la rivière et de ses affluents. La charge de fond est essentiellement composée de galets de schiste et dans une moindre mesure de quartzite. La nature de la roche mère confère à ces galets un aplatissement important. Les données hydrologiques utilisées dans cette étude proviennent de la station limnigraphique de Martelange (L5610, SPW-DGO3). Le débit à pleins bords $\left(Q_{b}\right)$ de la Sûre au niveau de cette station est de $32 \mathrm{~m}^{3} / \mathrm{s}$ et sa période de retour, calculée sur base des séries partielles, est de 0,8 an (Van Campenhout et al., 2020). Le module est de $3,8 \mathrm{~m}^{3} / \mathrm{s}$. Le taux de matières en suspension est faible $(<40 \mathrm{mg} / \mathrm{l}$ pour le débit à pleins bords). Cette valeur, relativement peu importante par rapport aux rivières de Wallonie (Van Campenhout et al., 2013) s'explique notamment par l'affectation du sol du bassin versant, principalement occupé par des prairies pour l'élevage bovin et par des résineux (Schmitz et Krippel, 2007). La densité de population dans les communes traversées par la Sûre est faible i.e., $<60$ habitants $/ \mathrm{km}^{2}$ (Direction générale Statistique - Statistics Belgium, 2011). Trois sites d'étude ont été sélectionnés par rapport à la présence d'U. crassus mise en évidence par les recensements réalisés par le DEMNA (fig. 1-2). Ces sites présentent une relative diversité dans leurs caractéristiques hydromorphologiques (fig. 2, tab. 1). La largeur à pleins bords $\left(w_{b}\right)$ varie de 8 à $15 \mathrm{~m}$ selon les sites. La pente locale est comprise entre $1,5 \%$ pour le site le plus en amont et 2,5\%o pour le site le plus en aval. Le site amont (site 1) est situé dans un fond de vallée étroit et boisé (UG7 et UG10 Natura 2000). La plaine alluviale du site 2 est plus large et constituée de prairies de fauche (UG2 et UG3 Natura 2000). Le site aval (site 3) est bordé par le versant en rive gauche et par une prairie pâturée en rive droite. L'impact anthropique est visible au niveau de ce secteur. Le site est situé à proximité d'habitations, et un petit barrage créant une zone d'écoulement lentique est présent à peu près $300 \mathrm{~m}$ en aval du début du site 3 . Le $\mathrm{D}_{50}$ de la charge de fond au niveau des sites d'étude est de l'ordre de $30 \mathrm{~mm}$, ce qui est inférieur au $\mathrm{D}_{50}$ mesuré au niveau de la station de Martelange située plus en aval (tab. 1).

Tab. 1 - Caractéristiques hydromorphologiques de la Sûre au niveau des sites d'étude et de la station limnigraphique de Martelange. Tab. 1 - Hydromorphological characteristics of the Sure River at study sites and at Martelange gauging station.

\begin{tabular}{|c|c|c|c|c|c|c|c|c|c|}
\hline & $\begin{array}{c}\text { Coordonnées } \\
\text { XY } \\
\text { (Lambert } \\
\text { belge 72) } \\
\end{array}$ & $\begin{array}{c}\text { Aire } \\
\text { de bassin } \\
\left(\mathbf{k m}^{2}\right)\end{array}$ & $D_{50}$ seuils & $D_{90}$ seuils & $\begin{array}{c}\text { Largeur à } \\
\text { pleins bords } \\
(W b)(\mathbf{m})\end{array}$ & $\begin{array}{c}\text { Puissance } \\
\text { spécifique } \\
\text { à pleins bords } \\
(\omega b)\left(\mathbf{w} / \mathbf{m}^{2}\right) \\
\end{array}$ & $\begin{array}{l}\text { Pente } \\
\text { locale } \\
(\%(\%)\end{array}$ & $\begin{array}{c}\text { Aire } \\
\text { cartographiée } \\
\left(\mathbf{m}^{2}\right)\end{array}$ & $\begin{array}{c}\text { Caractéristiques } \\
\text { particulières }\end{array}$ \\
\hline Site 1 & $\begin{array}{l}X: 242540 \\
Y: 64671\end{array}$ & 118 & 29 & 60 & 8,4 & 36 & 1,5 & 482 & 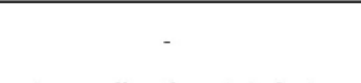 \\
\hline Site 2 & $\begin{array}{l}X: 243496 \\
Y: 64142\end{array}$ & 122 & 31 & 64 & 10 & 41 & 2 & 508 & $\begin{array}{l}\text { Présence d'un îlot végétalisé } \\
\text { créé suite à un recoupement de } \\
\text { méandre }\end{array}$ \\
\hline Site 3 & $\begin{array}{l}X: 245750 \\
Y: 61083\end{array}$ & 163 & 32 & 77 & 14,6 & 44 & 2,5 & 807 & $\begin{array}{l}\text { Présence d'un petit barrage } \\
\text { localisé } 268 \mathrm{~m} \text { en aval, } \\
\text { perturbant lécoulement }\end{array}$ \\
\hline $\begin{array}{c}\text { Station } \\
\text { Martelange }\end{array}$ & $\begin{array}{l}X: 248595 \\
Y: 58934\end{array}$ & 209 & 42 & - & 13,4 & 52 & 2,2 & $\mathrm{c}$ & Chenal canalisé en zone urbaine \\
\hline
\end{tabular}

Les valeurs du débit à pleins bords $\left(Q_{b}\right)$ au niveau des sites ont été extrapolées à partir des débits de la station limnigraphique de Martelange en utilisant la relation $\mathrm{q}=\mathrm{Q}(\mathrm{a} / \mathrm{A})^{0,8}$ où $\mathrm{q}\left(\mathrm{m}^{3} / \mathrm{s}\right)$ est le débit du site, $\mathrm{Q}\left(\mathrm{m}^{3} / \mathrm{s}\right)$ est le débit à la station limnigraphique, a $\left(\mathrm{km}^{2}\right)$ est la surface du bassin versant au niveau du site, et $\mathrm{A}\left(\mathrm{km}{ }^{2}\right)$ est la surface du bassin versant au niveau de la station (Bravard et Petit, 2000). Les pentes locales ont été calculées via le modèle numérique de terrain accessible sur le géoportail du Service public de Wallonie (SPW). Les données concernant la station limnigraphique de Martelange proviennent de Peeters et al. (2018).

The bankfull discharge $\left(Q_{b}\right)$ at study sites was extrapolated from discharge values recorded at the downstream gauging station of Martelange using the relation $q=Q(a / A)^{0.8}$ where $q$ $\left(\mathrm{m}^{3} \mathrm{~s}\right)$ is the discharge at the site, $Q$ is the discharge at the gauging station, a $\left(\mathrm{km}^{2}\right)$ is the catchment area at the site, and A $\left(\mathrm{km} \mathrm{m}^{2}\right)$ is the catchment area at the gauging station (Bravard and Petit, 2000). Local slopes were calculated by digital elevation model which is available on the geoportal of the Public Service of Wallonia (SPW). Data describing Martelange gauging station are from Peeters et al. (2018). 
Cette valeur a été obtenue en effectuant des granulométries selon la méthode d'échantillonnage proposée par Wolman (1954) sur les seuils amont et aval des secteurs, sauf dans le cas du site 3 où seul un seuil (à l'amont du secteur) a été échantillonné vu la présence en aval du barrage (profondeur trop importante, et faciès modifié). Ces trois sites d'étude, situés sur le cours principal de la Sûre, sont localisés en aval de la pollution de 2014, et se trouvent dans la masse d'eau ML12R évaluée dans un état écologique "bon " et ciblée pour atteindre l'objectif «très bon état » à l'horizon 2021 d'après la Directive Cadre sur l'Eau (2000/60/CE).

\section{Matériel et méthodes}

\subsection{Caractérisation des microhabitats d'U. crassus}

\subsubsection{Bathymétrie, mesure des vitesses d'écoulement et recensements}

Afin de caractériser les microhabitats aquatiques d'U. crassus, deux paramètres ont été cartographiés sur les trois sites d'étude : la topographie du fond du lit afin de connaître la profondeur et la vitesse du courant. Cette approche est basée sur la méthode proposée par Souchon et al. (1989) pour quantifier la capacité d'accueil d'un secteur de cours d'eau pour les poissons. Ces variables hydrauliques (profondeur et vitesse du courant) sont considérées comme de bons descripteurs des conditions d'habitat (Lamouroux et al., 2017). La méthode utilisée dans la présente étude est inspirée de celle appliquée pour le suivi scientifique de secteurs de cours d'eau restaurés dans le cadre du projet LIFE Walphy (Castelain et al., 2018). En pratique, la topographie du lit est levée au moyen d'une station totale (Leica TC600). Des profils transversaux ont été réalisés avec une interdistance maximale de 0,2 fois la largeur à pleins bords $\left(w_{b}\right)$, ce qui correspond à $2 \mathrm{~m}$ maximum. La longueur des tronçons cartographiés est de $6 w_{b}$ en moyenne. Le plan d'eau est également levé à la station totale. À chaque point topographique pris dans le lit mineur, la vitesse du courant est mesurée grâce à un courantomètre (Flo-Mate 2000). Cette mesure est réalisée à $40 \%$ de la hauteur d'eau depuis le fond car elle correspond à la vitesse moyenne du profil vertical (Bravard et Petit, 2000). L'interpolation des points topographiques et des vitesses est exécutée dans le logiciel ArcGis (ArcMap 10.5.1) selon la méthode Natural Neighbor, alors que celle des points du plan d'eau est réalisée via la méthode Inverse distance weighted. La profondeur est calculée en soustrayant l'interpolation de la topographie du lit à celle du plan d'eau. Les résultats sont ensuite stockés sous format raster de cellules de $0,1 \mathrm{~m}$. Les relevés microhabitats se sont déroulés entre le 5/03/2018 et le 20/04/2018 (fig. 3) avec un débit variant entre 0,5 et 0,7 fois le module à la station de Martelange, ce qui correspond à des conditions proches de la médiane des débits annuels ( $\mathrm{P} 50=1,9 \mathrm{~m}^{3} / \mathrm{s}$ ).

Les mulettes épaisses ont été recensées visuellement à l'aide d'aquascopes lorsque les conditions météorologiques et hydrologiques étaient optimales pour la détection de l'espèce (température $>15^{\circ} \mathrm{C}$, faible turbidité). Aucun événement morphogène ne s'est produit entre les relevés des microhabitats et les recensements des mulettes épaisses (fig. 3). L'emplacement exact des individus a été levé à la station totale de manière à les positionner par rapport à la répartition des profondeurs et vitesses de courant dans le secteur. Les recensements ont été effectués avec un débit variant de 0,2 à 0,3 fois le module à la station de Martelange. La longueur et la largeur de chaque individu ont été mesurées à l'aide d'un pied à coulisse. Lorsque plusieurs individus étaient proches les uns des autres (jusqu'à $15 \mathrm{~cm}$ ) un seul point a été levé pour le groupe. Les secteurs étudiés ont été fouillés de manière exhaustive (visuellement et manuellement dans les sédiments fins), il n'est cependant pas exclu que certains individus n'aient pas été détectés lors du recensement car enfouis dans la souscouche (Zieritz et al., 2014).

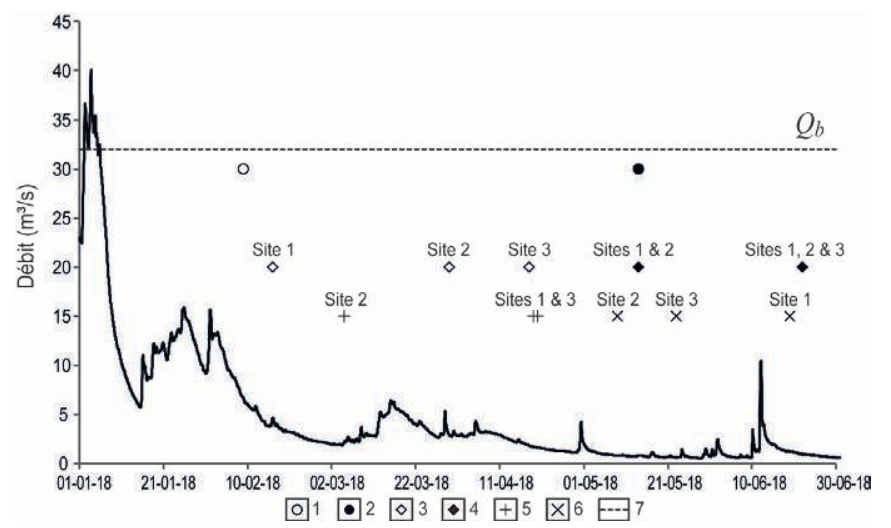

Fig. 3 - Hydrogramme de la période d'étude avec les dates des relevés.

1. Déploiement des galets marqués ; 2. Premier relevé de la position des galets marqués ; 3 . Installation des pièges à sédiments fins; 4 . Relevés des pièges à sédiments fins; 5 . Relevés de microhabitats; 6 . Recensements des individus Unio crassus; 7. Débit à pleins bords $\left(Q_{b}\right)$ au niveau de la station limnigraphique de Martelange.

Fig. 3 - Hydrograph for the study period with dates of surveys.

1. Deployment of the marked pebbles; 2. First survey of the marked pebbles location; 3 . Sediment samplers installation; 4. Sediment samplers surveys; 5 . Microhabitat surveys; 5. Population inventory of $U$. crassus; 7. Bankfull discharge $\left(Q_{b}\right)$ at the gauging station of Martelange.

\subsubsection{Importance relative des variables et préférences d'U. crassus}

Dans le but de déterminer leur importance dans la répartition spatiale d'U. crassus, la vitesse du courant et la profondeur ont été testées comme variables explicatives de la présence/absence des individus via un modèle logistique. La distance à la berge la plus proche a également été examinée en tant que variable explicative. En effet, nombre d'individus sont observés près des berges et de plus, les femelles $U$. crassus sont connues pour remonter les berges vers la surface de l'eau pour y émettre des jets contenant leurs glochidies (stade larvaire) (Vicentini, 2005). Cette distance à la berge correspond à la distance la plus proche entre l'individu et la limite du plan d'eau. Les cartes de bathymétrie et de répartition des vitesses ont été traitées comme un maillage de surfaces de $0,01 \mathrm{~m}^{2}$, chaque maille ayant une valeur de vitesse et de profondeur. Les données d'absence utilisées dans le modèle ont été extraites des rasters de répartition des vitesses de courant et de profondeurs. Cette technique de génération de pseudo-absences est couramment utilisée lorsque les données d'absence sont indisponibles (Engler, 2004). Toutes les analyses statistiques ont été réalisées avec le logiciel RStudio (version 1.2.5001).

La préférence des mulettes épaisses pour les microhabitats a été examinée en calculant un indice de préférence d'habitat (suitability index). Cet indice a été calculé pour la profondeur ainsi que pour 
la vitesse suivant la méthode utilisée par Hastie et al. (2000), et initialement proposée par Bovee (1986). La première étape consiste à mettre en rapport l'habitat utilisé et l'habitat disponible, c'est-à-dire calculer l'utilisation proportionnelle des mulettes de chaque classe d'habitat (classes de profondeurs et de vitesses). Ensuite l'indice de préférence d'habitat $(S I)$ est obtenu en divisant l'utilisation proportionnelle par l'utilisation proportionnelle maximale. Cette méthode fournit une valeur de 0 à $1 ; 0$ indiquant un habitat non adéquat et 1 caractérisant un habitat optimal.

\subsubsection{Analyse du substrat}

Des granulométries du substrat ont été réalisées sur des échantillons prélevés à l'emplacement des individus. Après déplacement des individus, la partie supérieure du lit était raclée sur une épaisseur de $10 \mathrm{~cm}$ au moyen de seaux à couvercles (10 1). Au total, sur les trois sites, 37 échantillons représentant soit un individu soit un groupe d'individus ont été prélevés. La fraction grossière $(>500 \mu \mathrm{m})$ a été tamisée, et pesée, par pas de 0,5 phi. La fraction inférieure à $500 \mu \mathrm{m}$ a été analysée avec un granulomètre à diffraction laser (Mastersizer 2000). Les résultats de ces analyses ont ensuite été traités avec le programme GRADISTATv8 (Blott et Pye, 2001) qui permet d'obtenir une représentation des échantillons dans un diagramme textural en triangle selon la classification de Folk et Ward (Folk, 1954). De plus, afin de déterminer les processus responsables de la mise en place des dépôts et de caractériser les faciès sédimentaires, les observations ont été reportées sur un diagramme CM de Passega $(1957,1964)$. Étant donné que de nombreux paramètres spécifiques à chaque cours d'eau influencent les caractéristiques de l'Image $\mathrm{CM}$ (position des segments), une Image établie pour des rivières ardennaises a été utilisée (Houbrechts et al., 2013).

\subsection{Colmatage du substrat et mobilité de la charge de fond}

Le colmatage physique de la sous-couche du lit de la rivière correspond à l'accumulation et l'infiltration de sédiments fins dans le substrat caillouteux (Datry et al., 2008). Ce phénomène provoque la réduction des échanges, notamment d'oxygène, entre la rivière et la sub-surface. Différentes techniques sont possibles pour caractériser l'infiltration de sédiments fins dans le lit des rivières (Bunte et Abt, 2001 ; Duerdoth et al., 2015). Parmi ces techniques, l'utilisation de dispositifs piégeant les sédiments fins est utilisée depuis plusieurs décennies (Frostick et al., 1984 ; Wood et Armitage, 1999). Les pièges à sédiments permettent en effet de quantifier l'infiltration de la charge fine dans la sous-couche graveleuse, et ainsi d'estimer le colmatage du substrat. Dans cette étude, un nouveau type de piège à sédiments fins a été développé, plus aisé à mettre en place et à relever. Il s'agit de cylindres $(110 \times 300 \mathrm{~mm})$ constitués de tuyaux en PVC (fig. 4). Ce dispositif présente une ouverture couverte d'un grillage métallique de maille de $5600 \mu \mathrm{m}$ d'une surface de $200 \mathrm{~cm}^{2}$ en tenant compte de la courbure du cylindre. Le cylindre, muni d'un bouchon à visser sur une des extrémités, doit être rempli de graviers autochtones de taille supérieure à la maille de la grille. La porosité du piège varie entre 45 et $55 \%$. Le piège est finalement posé perpendiculairement au courant sur un réceptacle hémicylindrique. Ce dernier est préalablement enchâssé dans le lit et fixé par des piquets métalliques, à une profondeur d'une dizaine de $\mathrm{cm}$, de manière à ce que le sommet du piège coïncide avec la surface du lit. Ce dispositif est particulièrement adapté pour des rivières à puissance modérée $\left(<50 \mathrm{~W} / \mathrm{m}^{2}\right)$. Le piège recueille ainsi les grains de taille inférieure à $5600 \mu \mathrm{m}$ mobilisés par le courant du cours d'eau et susceptibles de se déposer sur le fond ou de s'infiltrer. Lors de la récupération du dispositif à la fin de la période de suivi, un couvercle amovible est posé sur le grillage afin d'éviter la perte de sédiments fins par fluage. Le contenu des pièges est directement transvasé dans un seau pour être analysé en laboratoire. La fraction $>500 \mu \mathrm{m}$ est tamisée (par pas de 0,5 phi) et la partie $<500 \mu \mathrm{m}$ est analysée par granulométrie à diffraction laser. Après séchage, la fraction $>5600 \mu \mathrm{m}$ ayant servi à remplir les pièges est pesée. Cette masse est ensuite divisée par la densité $(2,6)$ pour connaître le volume des éléments grossiers (> $5600 \mu \mathrm{m}$ ). La part de vide est estimée par différence au volume du cylindre $\left(2851 \mathrm{~cm}^{3}\right)$. Enfin, de la même manière le volume de chaque classe granulométrique < $5600 \mu \mathrm{m}$ peut être calculé, ce qui permet d'estimer le pourcentage de remplissage des vides par les différentes fractions. La standardisation des données (sur une période de 30 jours) est nécessaire pour comparer les résultats des pièges entre eux car ils restent parfois en place pendant des périodes de durée différente, notamment lorsque les conditions hydrologiques ne permettent pas le relevé dans de bonnes conditions. Un piège a été installé sur chaque site au niveau d'un seuil. Ils sont restés en place entre le 16/02/2018 et le 22/06/2018. Le choix d'installer les dispositifs

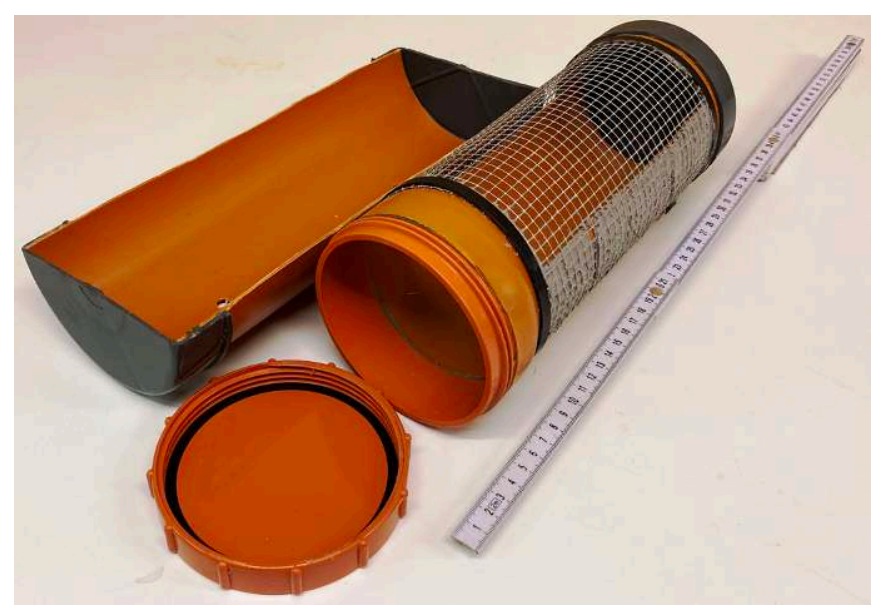

Fig. 4 - Illustration d'un piège à sédiments fins vide et de son réceptacle. Fig. 4 - Illustration of an empty fine sediment sampler and its receptacle.

sur les seuils a été fait dans un souci de représentativité du transport sédimentaire à l'échelle de la rivière, bien que les mulettes épaisses y soient trouvées en moindre nombre ou sous la couche d'armurage (Zając et Zając, 2011).

Parallèlement aux pièges, des sticks hypoxie ont été mis en place afin d'évaluer le degré de colmatage de la sous-couche. Les sticks hypoxie permettent de mettre en évidence des zones anoxiques dans le substrat du lit (Marmonier et al., 2004). Ces bâtonnets de pin non traités de $30 \mathrm{~cm}$ de longueur sont enfoncés dans les sédiments du lit. Lorsqu'il y a activité de bactéries anaérobies un noircissement du bois peut être observé. Les sticks hypoxie ont 
été installés transversalement dans les seuils en amont et/ou en aval direct des secteurs étudiés.

Le suivi à long terme de galets marqués dans la rivière permet d'identifier les débits mobilisateurs ainsi que la distance de mobilisation de la charge de fond caillouteuse, en relation notamment avec la puissance spécifique (Houbrechts et al., 2015). Afin d'identifier ces débits mobilisateurs, et d'en estimer l'impact sur $U$. crassus, des galets équipés de transpondeurs passifs à basse fréquence (passive integrated transponders, PIT Tags) ont été utilisés (Houbrechts et al., 2015 ; Piégay et al., 2016). Ces galets marqués ont été déployés le 09/02/2018 en aval du site 2. Par la suite, deux relevés de leur position en parcourant le cours d'eau à pied muni d'une antenne mobile ont été effectués ; le premier en mai 2018 et le second en mai 2019. En outre, des chaînes d'érosion ont été utilisées pour estimer l'épaisseur de la couche active de charriage du lit mineur (Laronne et al., 1994 ; Houbrechts et al., 2012). Une chaîne d'érosion consiste en une chaîne en acier de $30 \mathrm{~cm}$ dont le premier maillon coulissant est enchâssé dans une barre métallique enfoncée dans le lit. Cette configuration permet à la chaîne de descendre le long de la barre lors de l'incision du lit. L'accumulation de sédiments sur la chaîne en décrue permet alors de déterminer s'il y a incision, aggradation ou stabilité du lit. Les dispositifs ont été installés dans des seuils du site 1 et 2 .

\section{Résultats}

Pour chaque secteur, la cartographie des vitesses d'écoulement et de la bathymétrie, couplée à un recensement exhaustif de la population de mulettes épaisses, a permis de mettre en évidence leurs préférences pour des conditions de débits inférieures au module $\left(<0,1 Q_{b}\right)$. L'utilisation d'un modèle logistique a permis de tester l'influence de ces 2 variables, ainsi que de la proximité à la berge, et de leurs interactions sur la présence de l'espèce. Le substrat utilisé par $U$. crassus dans la Sûre a été caractérisé en fonction de ses indices granulométriques principaux $\left(D_{50}, D_{90} D_{99}\right)$, de sa teneur en différentes fractions granulométriques (gravier, sable, argilelimon), et des conditions de sédimentation et de transport de ces matériaux. Finalement, différents dispositifs ont permis de suivre et de caractériser le transport sédimentaire dans les secteurs à mulettes épaisses.

\subsection{Recensements d'U. crassus}

Au total, 131 individus $U$. crassus ont été trouvés sur les 3 sites. Les recensements ont été réalisés postérieurement aux relevés topographiques ainsi certains individus ont été trouvés en dehors des zones cartographiées. Au total, 107 individus sont pris en compte dans les analyses statistiques; 12 individus pour le site $1\left(482 \mathrm{~m}^{2}\right), 88$ individus pour le site $2\left(508 \mathrm{~m}^{2}\right)$, et 7 individus pour le site $3\left(807 \mathrm{~m}^{2}\right)$. La distribution des tailles des individus entre les 3 sites est homogène. La longueur des coquilles renseigne sur l'âge des individus grâce à une relation établie par Dunca (2014) pour les populations de la Sûre. Selon cette courbe de croissance, il apparait que $80 \%$ des individus ont entre 6 et 14 ans. Les juvéniles (caractérisés par une coquille mesurant jusqu’à $35 \mathrm{~mm}$ ) représentent $6 \%$ des individus recensés (fig. 5).

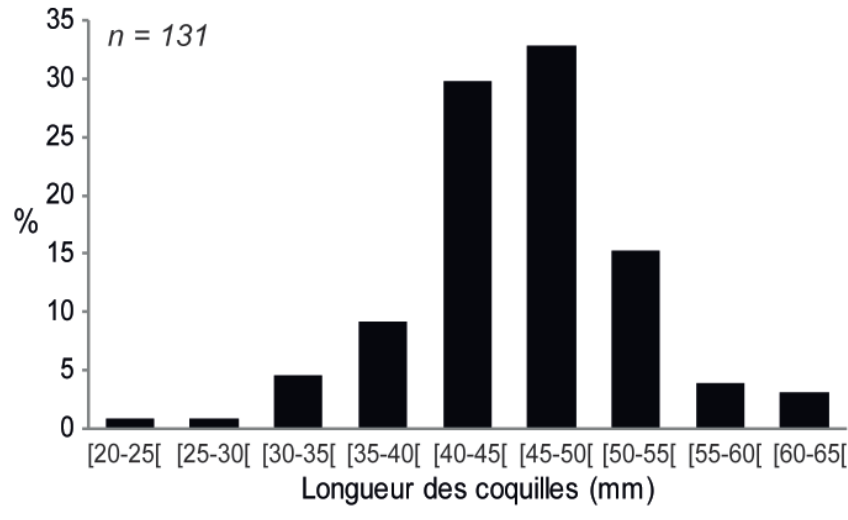

Fig. 5 - Distribution des tailles des individus pour les 3 sites d'étude confondus.

Fig. 5 - Shell lengths distribution for the 3 study sites altogether.

\subsection{Préférences d'habitats}

Il n'existe pas de différence significative entre les sites en ce qui concerne la répartition d'U. crassus dans les différentes classes de profondeur (Test exact de Fisher : $p$-value $=0,64$ ) et de vitesse (Test exact de Fisher : $p$-value $=0,10$ ). Lors des recensements, les mulettes se situaient majoritairement en dehors des seuils, et en dehors des mouilles (fig. 6). Elles étaient plus fréquemment observées dans les dépôts latéraux ou dans la convexité de méandres. La distance moyenne entre leur position et la berge la plus proche est de $2 \mathrm{~m}$. Elles ont été recensées dans des profondeurs variant entre 0,16 et $0,89 \mathrm{~m}$, à l'exception d'une mulette qui a été trouvée à l'interface eau-air enchâssée dans le limon compact de la berge (site 2). La profondeur maximale mesurée est de 1,3 m (site 3). La plupart des individus (74\%) étaient localisés dans des zones de profondeurs intermédiaires, entre 0,4 et $0,8 \mathrm{~m}$ (fig. 7). Ces classes de profondeur, de 0,4 à $0,6 \mathrm{~m}$ et de 0,6 à $0,8 \mathrm{~m}$, sont également les plus fréquentes. L'indice de préférence d'habitat (SI) tend à indiquer que ce sont aussi les classes les plus adéquates pour les individus. Le $S I$ de la classe de $<0,2$ m indique une qualité élevée malgré le nombre peu important d'individus recensés dans ces profondeurs.

La vitesse maximale a été mesurée au niveau du site 2 avec 1,6 m/s ; les vitesses maximales mesurées au site 1 et 2 étaient de $1 \mathrm{~m} / \mathrm{s}$ et de $0,6 \mathrm{~m} / \mathrm{s}$ respectivement. Les individus ont été trouvés dans des zones de vitesses de courant comprises entre 0 et $0,89 \mathrm{~m} / \mathrm{s}$. La majorité des individus ( $87 \%$ ) a été observée dans des zones de vitesses comprises entre 0 et $0,4 \mathrm{~m} / \mathrm{s}$ (fig. 8). Les vitesses de courant de 0 à $0,4 \mathrm{~m} / \mathrm{s}$ sont également les plus disponibles. La courbe de préférence indique que ces vitesses seraient les plus adéquates pour $U$. crassus, et que les vitesses jusqu'à $1 \mathrm{~m} / \mathrm{s}$ ne gêneraient pas l'espèce. À noter qu'aucune corrélation significative n’a été trouvée entre la taille des coquilles et les profondeurs ou les vitesses de courant.

\subsection{Importance relative des variables environnementales}

Le rôle relatif des variables dans la présence de l'espèce a été déterminé via un modèle logistique. Le meilleur modèle a été sélectionné sur base de l'AIC (Akaike information criterion) le plus petit (AIC = 207,86). L'AUC (area under the curve), qui renseigne sur la capacité prédictive du modèle, est de 0,82. Les odds-ratio 

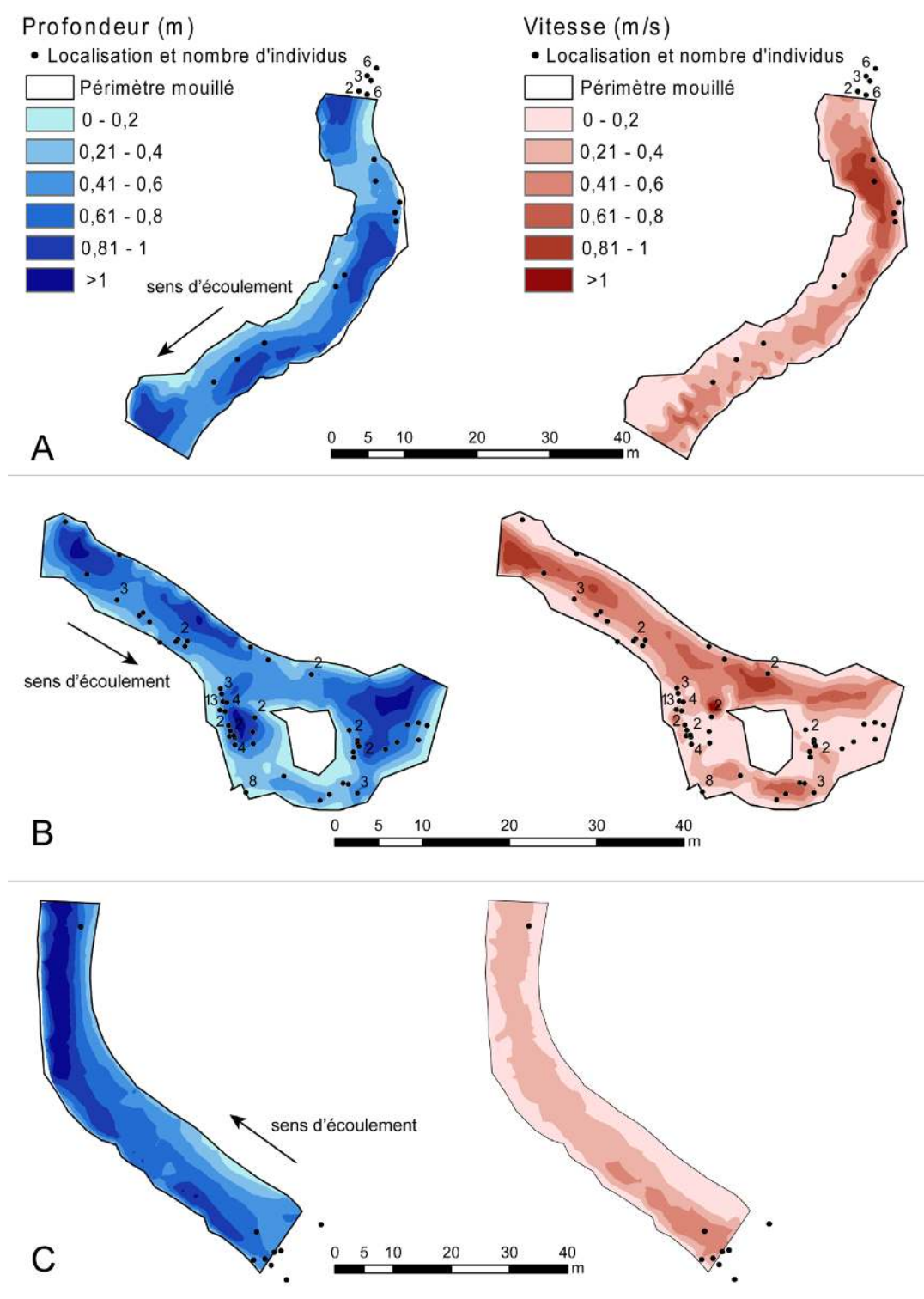

Fig. 6 - Cartes de bathymétrie et de répartition des vitesses de courant des 3 sites d'étude avec localisation des individus.

A : bathymétrie et vitesses de courant au site 1 ; B : bathymétrie et vitesses de courant au site 2 ; C: bathymétrie et vitesses de courant au site 3 . Les points de localisation d'individus sans chiffre représentent un seul individu.

Fig. 6 - Bathymetric and flow velocity maps at the 3 study sites with individuals location.

$A$ : bathymetry and flow velocity at site $1 ; B$ : bathymetry and flow velocity at site $2 ; C:$ bathymetry and flow velocity at site 3 . Location dots without numbers represent one individual.

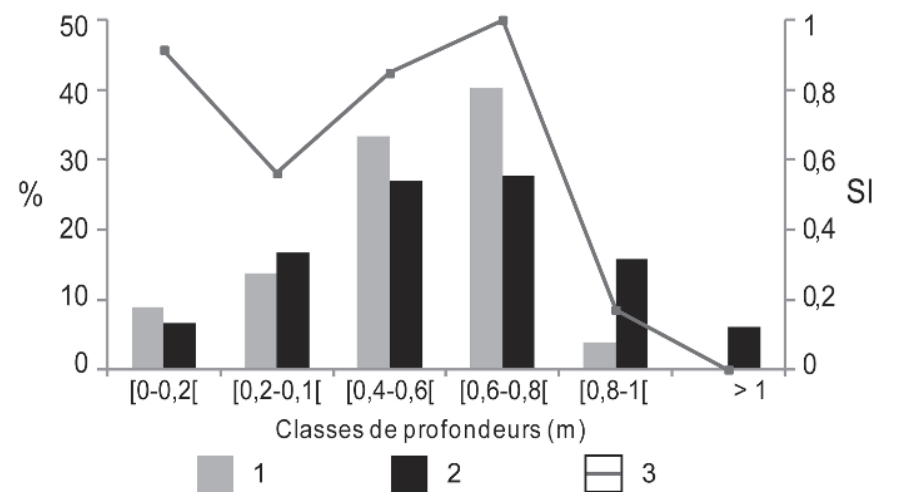

Fig. 7 - Disponibilité des différentes classes de profondeur (en \%), utilisation de ces classes par $U$. crassus (en \%), et indice de préférence d'habitat (SI) pour les 3 sites confondus.

1. Utilisation de l'habitat par les mulettes épaisses ; 2. Disponibilité de l'habitat ; 3. Indice de préférence d'habitat (SI).

Fig. 7 - Depth class availability (\%), use by U. crassus of those classes (\%), and suitability index (SI) for the 3 study sites altogether.

1. Habitat use by the thick-shelled river mussels; 2. Habitat availability; 3. Suitability index (SI).

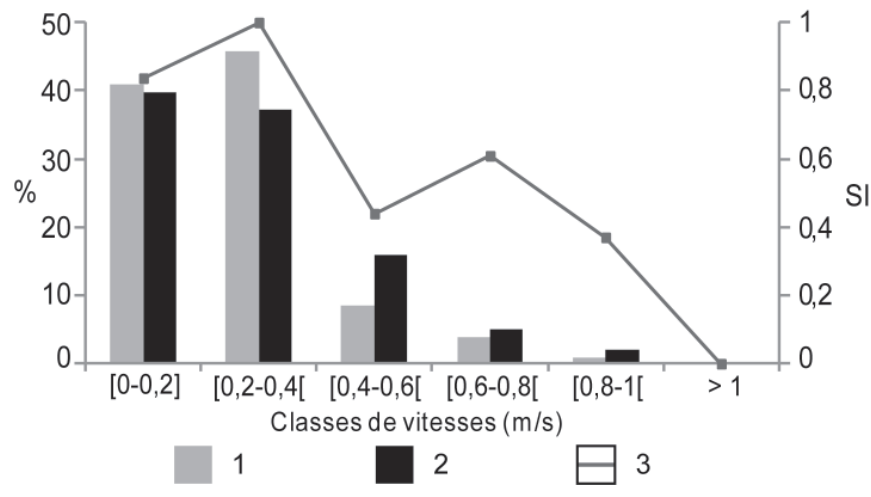

Fig. 8 - Disponibilité des différentes classes de vitesses (en \%), utilisation de ces classes par U. crassus (en \%), et indice de préférence (SI) pour les 3 sites confondus.

1. Utilisation de l'habitat par les mulettes épaisses ; 2. Disponibilité de l'habitat ; 3 . Indice de préférence d'habitat (SI).

Fig. 8 - Availability of flow velocity classes (\%), use by U. crassus of those classes (\%), and suitability index (SI) for the 3 study sites altogether.

1. Habitat use by the thick-shelled river mussels; 2. Habitat availability; 3. Suitability index (SI). 
$(O R)$, qui renseignent sur la force de l'association entre les variables environnementales et l'occurrence de l'espèce, sont présentés avec leur intervalle de confiance (fig. 9). Un odd-ratio largement supérieur à 1 indique que lorsque la variable augmente, la probabilité de trouver un individu $U$. crassus augmente elle aussi ; au contraire un odd-ratio $<1$ indique que la probabilité de présence diminue lorsque la variable augmente. La présence des termes d'interaction dans le meilleur modèle indiquent une synergie entre certaines variables; entre la distance à la berge la plus proche et la profondeur, et entre la distance à la berge la plus proche et la vitesse de courant. Les profondeurs influencent particulièrement la présence/absence de l'espèce : tandis que la probabilité de trouver un individu augmente avec la hauteur d'eau, cette tendance s'inverse lorsque la profondeur est trop importante (cf. f2 profondeur, $O R$ de la profondeur élevée à la puissance 2) (fig. 9). L'augmentation de la vitesse de courant semble avoir un impact négatif sur la probabilité de présence. La distance à la berge montre l'impact le plus limité sur la présence/ absence de l'espèce. Toutefois, cette analyse ne porte que sur une rivière relativement étroite $(<15 \mathrm{~m})$.

\subsection{Analyse granulométrique du substrat}

Les 37 échantillons prélevés où se trouvaient des moules sont caractérisés en fonction de leurs pourcentages respectifs en gravier $(64-2 \mathrm{~mm})$, en sable $(2000-63 \mu \mathrm{m})$ et en fraction argilo-

\begin{tabular}{|c|c|c|}
\hline Variables & Odds ratio & $p$-valeur \\
\hline profondeur & $1 \mapsto$ & $<0,001$ \\
\hline f2 profondeur & $\longmapsto \quad$ I & $<0,001$ \\
\hline vitesse & $\sim_{1}^{\prime}$ & 0,005 \\
\hline distance & 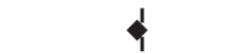 & 0,018 \\
\hline f2 distance & 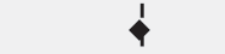 & $<0,001$ \\
\hline distance*profondeur & $1 *$ & $<0,001$ \\
\hline distance*vitesse & 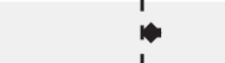 & 0,006 \\
\hline (Intercept) & 4 & 0,169 \\
\hline
\end{tabular}

Fig. 9 - Valeurs des odds-ratio avec leur p-valeur pour chaque variable.

Les variables retenues dans le meilleur modèle sont la profondeur, la profondeur à la puissance 2, la vitesse du courant, la distance à la berge, la distance à la berge à la puissance 2, la distance à la berge en interaction avec la profondeur, et distance à la berge en interaction avec la vitesse du courant.

Fig. 9 - Odds ratio with their p-value for each variable.

Variables kept in the best model are water depth, water depth to the power of 2, flow velocity, distance to the nearest bank, distance to the nearest bank to the power of 2 , distance to the nearest bank in interaction with depth, distance to the nearest bank in interaction with flow velocity.

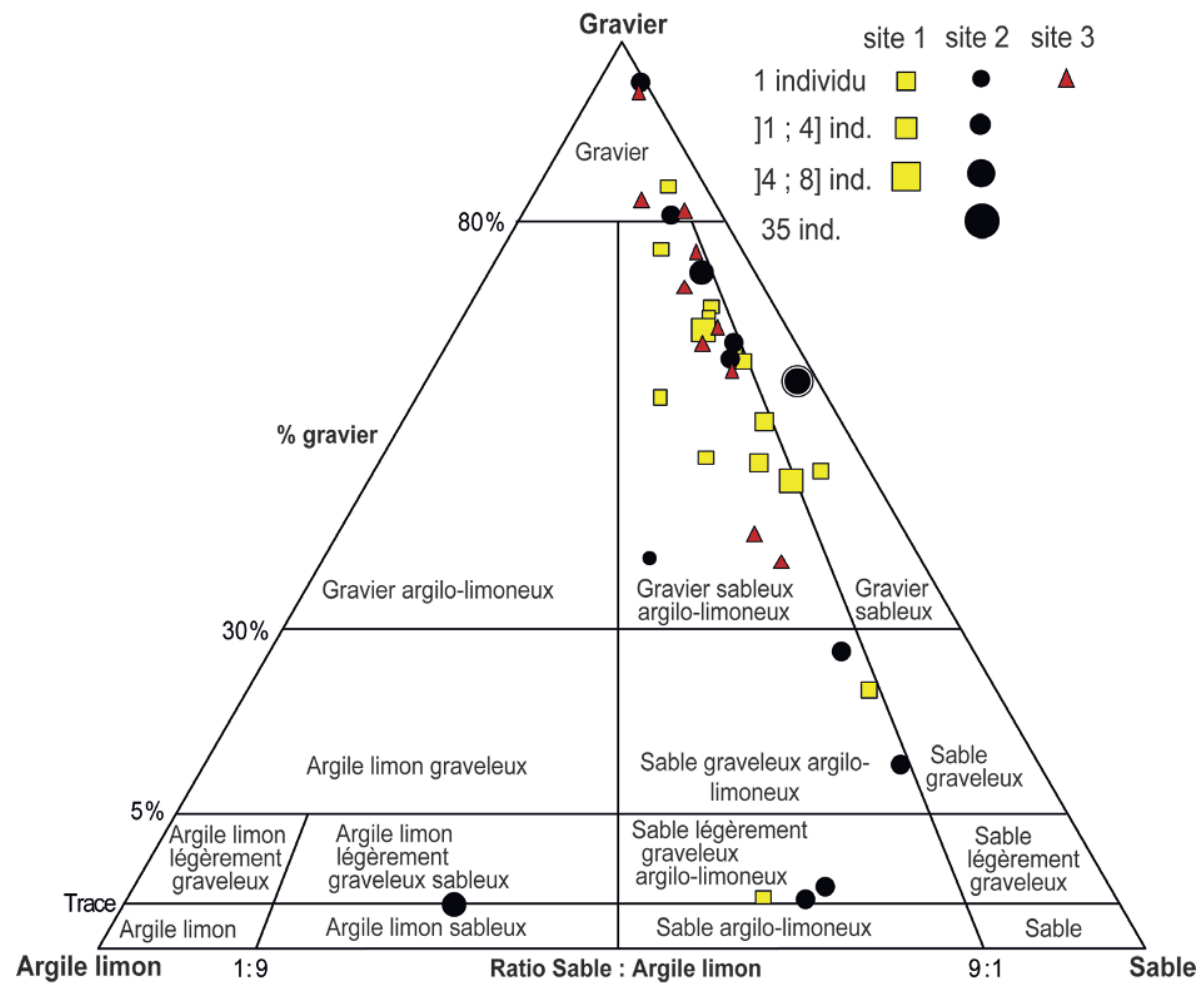

Fig. 10 - Classification des échantillons de substrat habité par $U$. crassus selon leurs pourcentages en gravier, sable et argilelimon.

Diagramme textural réalisé avec GRADISTATv8 (Blott et Pye, 2001).

Fig. 10 - Classification of substrate samples inhabited by U. crassus according to their percentages of gravel, sand and mud.

Textural diagram obtained with GRADISTATv8 (Blott and Pye, 2001). limoneuse $(<63 \mu \mathrm{m})$ (fig. 10). Les échantillons de substrat analysés se répartissent en 6 types : gravier, gravier sableux, gravier sableux argilo-limoneux, sable graveleux argilo-limoneux, sable légèrement graveleux argilo-limoneux, et fraction argilo-limoneuse légèrement graveleuse sableuse. La classe majoritaire, gravier sableux argilolimoneux, contenait $41 \%$ des individus recensés. $31 \%$ des individus se situaient dans du gravier sableux, classe qui correspond à $5 \%$ des échantillons (2 prélèvements) car de nombreux individus s'y trouvaient en groupe. La médiane pondérée des $D_{50}$ des échantillons est de 2,6 mm (P25 = 2,0; P75 = 4,6), la médiane pondérée des $D_{90}$ est de $11,3 \mathrm{~mm}(\mathrm{P} 25=7,3 ; \mathrm{P} 75=23,1)$, et la médiane pondérée des $D_{99}$ est de 25,9 mm (P25 = 17,6; P75 = 31,3). Aucune corrélation statistiquement significative entre les indices granulométriques des échantillons $\left(D_{50}\right.$ et $\left.D_{99}\right)$ et la taille des individus (longueur et 
Tab. 2 - Composition des échantillons en gravier, sable et argile-limon exprimée en pourcentages.

Tab. 2 - Sample contents of gravel, sand and mud (clay and limestone) expressed as percentages.

\begin{tabular}{lccc}
\hline & \% gravier & \% sable & \% argile-limon \\
\hline Site 1 & 58 & 34 & 8 \\
\hline Site 2 & 50 & 37 & 13 \\
\hline Site 3 & 70 & 23 & 7 \\
\hline
\end{tabular}

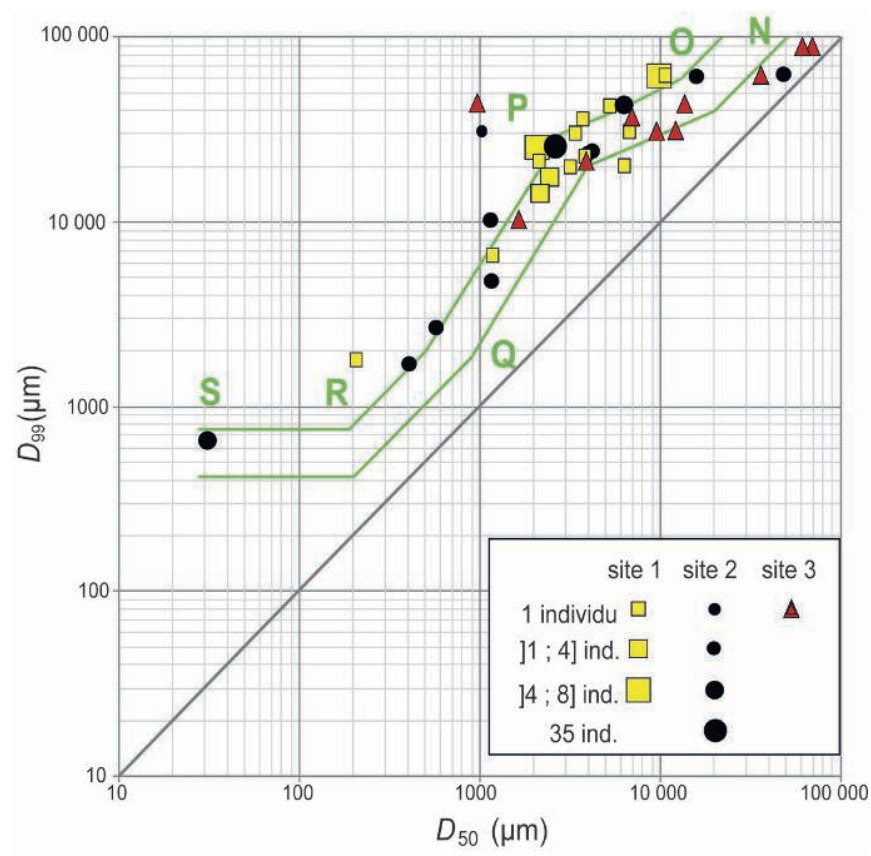

Fig. 11 - Diagramme théorique de Passega des rivières ardennaises à lit caillouteux (d'après Houbrechts et al., 2013), avec les échantillons de substrat habités par U. crassus.

La diagonale correspond à l'égalité entre la médiane $\left(D_{50}\right)$ et le percentile le plus grossier $\left(D_{99}\right)$ ( = droite de tri parfait). Segment SR : dépôt issu de la suspension uniforme. RQ : dépôt résultant du ralentissement des courants turbulents, c'est-à-dire de la suspension gradée lorsque la charge dépasse la capacité de transport. QP : dépôt de suspension gradée avec début de roulage (compétence encore trop faible). PO : dépôt mis en place par roulage avec toujours un peu d'éléments issus de la suspension. ON : dépôt formé par roulage, courant trop important pour permettre aux éléments fins de se déposer.

Fig. 11 - CM diagram of Ardenian gravel-bed rivers (Houbrechts et al., 2013) with substrate samples inhabited by U. crassus.

Diagonal shows equality between the median $\left(D_{50}\right)$ and the $99^{\text {th }}$ percentile $\left(D_{90}\right)(=$ line of perfect sorting). SR segment : deposit from uniform suspension. RQ : deposit from turbulent currents slowing down that is from graded suspension when load is above transport capacity. QP : deposit from graded suspension with early rolling. $P O$ : deposit from rolling with still a bit of elements from suspension. $\mathrm{ON}$ : deposit from rolling.

largeur) n'a pu être mise en évidence (corrélation de Spearman).

La fraction fine $(<2000 \mu \mathrm{m})$ représentait en moyenne entre 30 et $50 \%$ des échantillons (tab. 2). À noter que les échantillons du site 2 présentaient une certaine variabilité entre eux (médiane pondérée des pourcentages : gravier $60 \%$, sable $35 \%$, argile-limon $5 \%$ ). Le pourcentage de matières organiques des échantillons était toujours $<0,3 \%$ sauf pour 2 échantillons où ce taux était de 3 et $1 \%$ (respectivement site 1 et 2 ).

Les 37 échantillons de substrat, représentés par leur $D_{50}$ et $D_{99}$, sont présentés dans une Image de Passega définie pour des rivières ardennaises (Houbrechts et al., 2013). Les mulettes sont présentes dans tous les types de dépôts, depuis la suspension uniforme jusqu’à ceux résultant du roulage (fig. 11). Il est intéressant de noter la plus forte concentration d'échantillons à la rupture entre le segment $\mathrm{QP}$ et le segment PO. L'échantillon du segment RS provient d'un dépôt issu de la suspension uniforme. Les sédiments de cet échantillon, prélevés en pied de berge, proviennent probablement d'un pan de berge (limon compact) qui s'est détaché, puis désagrégé, formant la zone de sédiments meubles qui a finalement été colonisée par 8 individus. De plus, cette zone située dans le bras droit de l'îlot du site 2, est soumise à des cellules de contre-courant, et les zones de faible courant qui en résultent sont propices à la sédimentation de limon.

\subsection{Colmatage du substrat et mobilité de la charge de fond}

Le remplissage des pièges à sédiments s'est avéré relativement faible, variant entre 3 et $11 \%$ par mois pour la fraction $<2000$ $\mu \mathrm{m}$ (tab. 3). Le pourcentage de vide initial des pièges était de $50 \%$ en moyenne. Le taux remplissage le moins important correspond au piège de la première période d'étude placé dans le site le plus en amont, i.e., site 1-1 (tab. 3). Cette période est caractérisée par un débit maximal de $6,4 \mathrm{~m}^{3} / \mathrm{s}\left(0,2 Q_{b}-16 / 03 / 2018\right)$. Lors de la deuxième période de suivi, caractérisée par un débit maximal de $10,4 \mathrm{~m}^{3} / \mathrm{s}\left(0,3 Q_{b}-12 / 06 / 2018\right)$, les pièges des sites 1 et 2 montrent les pourcentages de remplissage les plus importants. Le piège du site 3 , en place lors du débit maximal de $10,4 \mathrm{~m}^{3} / \mathrm{s}$, présente un taux de remplissage inférieur à ceux des sites 1 et 2 . Le peu de matière organique piégée consistait en des macro-débris végétaux. Les sticks hypoxie ont mis en évidence des différences de niveau de colmatage entre les seuils des 3 sites. Le seuil du site 2 n'était pas colmaté, celui du site 1 l'était modérément, et celui du site 3 était totalement colmaté (tab. 3).

Les distances de charriage relevées lors des deux campagnes de détection des galets marqués sont présentées dans le Tableau 4. Le premier relevé indique que la crue de $0,2 Q_{b}$ a entraîné une très faible mobilisation. De plus, la mobilisation des 6 galets a vraisemblablement été favorisée par un effet de saillie des éléments injectés. En effet, les distances parcourues observées lors du premier relevé ne sont généralement pas significatives car une réorganisation naturelle des galets par la rivière est nécessaire avant d'estimer la mobilisation effective de la charge de fond (Houbrechts et al., 2015). La proximité des galets les uns par rapport aux autres, provoquant des collisions de signaux, explique le taux de récupération limité du premier relevé. Le second relevé a été réalisé un an plus tard (05/2019) suite à 5 crues comprises dans l'intervalle 0,5 à $1,3 Q_{b}$, correspondant à une puissance spécifique comprise entre 19 et $51 \mathrm{~W} / \mathrm{m}^{2}$. L'axe b moyen des galets mobilisés durant cette période $(36 \mathrm{~mm})$ est légèrement supérieur au $D_{50}$ du lit $\left(D_{50}\right.$ seuils de $30 \mathrm{~mm}$ ) (tab. 1). La distance maximale (172 m) a été parcourue par un élément de $26 \mathrm{~mm}$ d'axe b, et la taille moyenne des éléments ayant parcouru les 5 distances maximales (136 à $172 \mathrm{~m}$ ) est de $29 \mathrm{~mm}$.

Les chaînes d'érosion installées dans le site 1 ont démontré qu'il n'y a pas eu d'aggradation ni d'incision du lit au niveau du seuil où elles étaient installées pour une crue maximum de $0,3 Q_{b}$ $\left(12 / 06 / 2018 ; 10,4 \mathrm{~m}^{3} / \mathrm{s}\right)$. Les chaînes installées dans le site 2 ont été perturbées par l'action de l'homme et ne peuvent donc pas fournir des résultats quantifiables. 
Tab. 3 - Résultats des pièges à sédiments et des sticks hypoxie.

Tab. 3 - Results from sediment samplers and hypoxia wooden stakes.

\begin{tabular}{|c|c|c|c|c|c|}
\hline & $\begin{array}{l}\text { Site } \\
1-1\end{array}$ & $\begin{array}{l}\text { Site } \\
1-2\end{array}$ & $\begin{array}{l}\text { Site } \\
2-1\end{array}$ & $\begin{array}{l}\text { Site } \\
2-2\end{array}$ & $\begin{array}{l}\text { Site } \\
3-1\end{array}$ \\
\hline $\begin{array}{l}\% \text { de vide initial } \\
\text { dans le piège }\end{array}$ & 53 & 50 & 55 & 49 & 45 \\
\hline $\begin{array}{l}\text { \% espace rempli } \\
\text { par }<5600 \mu \mathrm{m} / \text { mois }\end{array}$ & 4 & 14 & 11 & 13 & 8 \\
\hline $\begin{array}{l}\% \text { espace rempli } \\
\mathrm{par}<2000 \mu \mathrm{m} / \text { mois }\end{array}$ & 3 & 11 & 8 & 11 & 5 \\
\hline $\begin{array}{l}\% \text { espace rempli } \\
\text { par }<500 \mu \mathrm{m} / \text { mois } \\
\% \text { espace rempli }\end{array}$ & 2 & 9 & 4 & 7 & 4 \\
\hline $\begin{array}{l}\text { par la matière } \\
\text { organique/mois }\end{array}$ & 0,25 & 0 & 0,54 & 0 & 0 \\
\hline période (jours) & 87 & 39 & 45 & 39 & 63 \\
\hline $\begin{array}{l}\text { débit } \max \left(\mathrm{m}^{3} / \mathrm{s}\right) \\
\text { à Martelange }\end{array}$ & 6,4 & 10,4 & 4,3 & 10,4 & 10,4 \\
\hline pente locale $(\%)$ & \multicolumn{2}{|c|}{1,5} & \multicolumn{2}{|c|}{2} & 2,5 \\
\hline $\begin{array}{l}\text { profondeur moyenne } \\
\text { du début de l'hypoxie } \\
\text { depuis la surface des } \\
\text { sédiments }(\mathrm{cm})\end{array}$ & \multicolumn{2}{|c|}{15} & \multicolumn{2}{|c|}{25} & I \\
\hline
\end{tabular}

Site 1-1 : le premier chiffre fait référence à la désignation du site, le second chiffre à la période de relevé ; par exemple dans ce cas : premier relevé du piège du site 1 . Les pièges des sites 1 et 2 ont été relevés à 2 reprises; le piège du site 3 a été relevé une fois. Site 1-1 : first number refers to the study site denomination; the second one refers to the study period; for example here site 1-1 means "first collecting survey of the site 1 sediment sampler". Sites 1 and 2 sediment samplers were surveyed twice; site 3 sediment sampler was surveyed once.

Tab. 4 - Résultats du suivi des galets marqués.

Tab. 4 - Results of the marked pebbles monitoring.

\begin{tabular}{lcc}
\hline & $\begin{array}{c}\text { relevé 1 } \\
(\mathbf{0 5} / \mathbf{2 0 1 8})\end{array}$ & $\begin{array}{c}\text { relevé 2 } \\
(\mathbf{0 5} / \mathbf{2 0 1 9})\end{array}$ \\
\hline Taux de récupération (\%) & 10 & 73 \\
\hline \% de galets mobilisés & 4 & 65 \\
\hline Axe b moyen des galets mobilisés (mm) & 37 & 36 \\
\hline Plus gros galet mobilisé (mm) & 46 & 55 \\
\hline Distance moyenne parcourue $(\mathrm{m})$ & 2 & 24 \\
\hline Distance maximale parcourue $(\mathrm{m})$ & 3 & 172 \\
\hline$Q_{\max }\left(\mathrm{m}^{3} / \mathrm{s}\right)$ & 6,4 & 40,2 \\
\hline$Q_{\max } / Q_{b}$ & 0,2 & 1,3 \\
\hline Puissance spécifique $\omega Q_{\max }\left(\mathrm{W} / \mathrm{m}^{2}\right)$ & 8 & 51 \\
\hline
\end{tabular}

\section{Discussion}

\subsection{Structure populationnelle d'U. crassus}

Les individus recensés étaient globalement âgés de 6 ans ou plus, ce qui signifie que ce sont des survivants de la pollution de 2014. La répartition des tailles des coquilles mesurées indique une population adulte vieillissante (Zettler et Jueg, 2007). Cependant, étant donné que les juvéniles restent les premières années de leur vie dans le sédiment, leur détection est moins aisée (Lamand et Beisel, 2014). La localisation des jeunes mulettes nécessite en effet la plupart du temps de fouiller le substrat à la main ou au tamis (Zettler et Jueg, 2007). La présence de juvéniles, et donc la preuve que les populations sont toujours fonctionnelles, a été démontrée un an après cette étude lors d'une recherche exhaustive réalisée par les agents du Parc naturel Haute-Sûre Forêt d'Anlier. À cette occasion, le substrat du secteur en aval direct du site 3 a été fouillé systématiquement à la main. Lors de ce recensement, $40 \%$ des mulettes épaisses détectées étaient des juvéniles (longueur $<35 \mathrm{~mm}$ ). Il est donc extrêmement vraisemblable que la faible proportion de juvéniles recensés dans les secteurs étudiés soit due à leur détectivité limitée, et non une preuve de leur absence.

\subsection{Préférences hydrauliques}

La grande majorité des individus se situait dans des zones présentant une hauteur d'eau intermédiaire (entre 0,4 et $0,8 \mathrm{~m}$; pour des conditions de basses eaux inférieures au module). Le SI (indice de préférence) semble indiquer que les faibles profondeurs $(<0,2 \mathrm{~m})$ sont également adéquates pour l'espèce. La gamme de profondeurs où les mulettes épaisses ont été trouvées dans la Sûre concorde avec les valeurs de la littérature : entre 0,2 et $0,7 \mathrm{~m}$ (Fouillé, 2013), entre 0,2 et $0,4 \mathrm{~m}$ (Vicentini, 2005), entre 0,3 et $0,8 \mathrm{~m}$ (Engel et Wächtler, 1989). Néanmoins, ces mesures sont difficilement comparables sans informations sur les conditions hydrologiques des rivières étudiées. La hauteur d'eau s'est révélée statistiquement significative pour la présence/absence des individus. Les odds-ratio fournis par la modélisation logistique pour la profondeur et pour la profondeur élevée à la puissance 2 suggèrent une gamme optimale de hauteur d'eau. Jusqu'à un certain seuil, la profondeur et la probabilité de présence augmentent de concert mais lorsque ce seuil est dépassé, la probabilité de présence décroît lorsque la profondeur augmente. À noter que ces résultats peuvent être influencés par la technique de recensement.

En ce qui concerne les vitesses d'écoulement, les individus ont été principalement trouvés dans des zones de faible courant $(<0,4 \mathrm{~m} / \mathrm{s}$; pour des conditions de basses eaux inférieures au module). Cependant, des zones de courants plus rapides paraissent pouvoir convenir pour quelques individus comme le suggère le $S I$ de la classe $0,6-0,8 \mathrm{~m} / \mathrm{s}$ (fig. 8). Dans plusieurs cours d'eau du bassin du Danube supérieur, Stoeckl et Geist (2016) notent également la tolérance de l'espèce à une large gamme de conditions hydrologiques $(0,22$ à $0,95 \mathrm{~m} / \mathrm{s})$, ainsi que sa préférence marquée pour les eaux calmes $(<0,35 \mathrm{~m} / \mathrm{s})$. La régression logistique indique une influence de la vitesse dans la présence/ absence d'U. crassus. Le rôle joué par la vitesse d'écoulement dans la répartition des mulettes est cependant plus modéré que celui de la hauteur d'eau. Comme le suggère la valeur négative de l'odd-ratio, plus la vitesse augmente, plus la probabilité de trouver un individu diminue.

La capacité prédictive limitée du modèle provient du nombre réduit de variables utilisées et de leur nature. En effet, utilisées seules, les variables physiques simples comme la vitesse et la profondeur prédisent rarement efficacement la distribution (Steuer et al., 2008). Les valeurs de ces variables étant valables pour un débit donné, elles 
performent donc de manière restreinte pour d'autres conditions de débits (Morales et al., 2006). Néanmoins, la profondeur et la vitesse restent des facteurs importants qui limitent la distribution des moules pour des périodes de débits de base (Layzer et Madison, 1995), et la faible capacité prédictive n'influe pas sur la qualité du modèle. Cependant, étant donné les relations qui existent entre les variables (hauteur d'eau, vitesse, distance à la berge), leur contribution individuelle réelle dans la présence/absence d'U. crassus est incertaine.

\subsection{Interactions des variables}

Plusieurs éléments peuvent expliquer l'importance prépondérante de la profondeur et l'influence des autres variables dans la présence/ absence d'U. crassus. Premièrement, un faible niveau d'eau signifie une température de l'eau plus élevée ce qui entraine une moindre disponibilité en oxygène. Or, la qualité du contenu en oxygène dans l'eau semble être un facteur vital pour U. crassus (Douda, 2007). Une hausse de température provoque également une mortalité des glochidies plus importante (Jansen et al., 2001 ; Schneider et al., 2018). Deuxièmement, l'inclination des mulettes pour une gamme de profondeurs est à mettre en relation avec la vitesse du courant. En effet, la vitesse d'écoulement est fonction notamment de la hauteur d'eau (Leopold et al., 1964). Les espaces occupés préférentiellement par $U$. crassus présentent un courant d'eau relativement lent, mais non nul. Les zones colonisées pourraient ainsi être limitées par les seuils de conditions de débits requis pour le transport des nutriments, de l'oxygène et des déchets (Steuer et al., 2008). L'affinité pour des zones plus lentiques est aussi à corréler avec la capacité de filtration qui diminue lorsque la vitesse devient trop importante (Ackerman, 1999). Troisièmement, les vitesses de courant sont à mettre en relation avec la distance à la berge. En effet, dans les tronçons subrectilignes, les vitesses les plus élevées sont généralement mesurées au centre du cours d'eau alors que la vitesse décroit vers les berges (Leopold et al., 1964 ; Bravard et Petit, 2000). Ainsi l'importance relativement faible de la distance à la berge peut être expliquée par la répartition latérale des vitesses du courant (et des profondeurs) qui pourrait provoquer cette configuration spatiale souvent observée chez les Unionida (Box et al., 2002 ; Aldridge et al., 2007). Lors des recensements, il est effectivement apparu que les mulettes sont trouvées plus fréquemment près des berges où les vitesses et les profondeurs sont moins importantes qu'au centre du cours d'eau. Dans les zones plus sinueuses, les mulettes épaisses sont également réparties dans différentes zones d'accumulation de sédiments. Les mouvements latéraux des mulettes vers les berges observés dans différents cas auraient donc pour but de se placer dans une zone de profondeur optimale (Schwalb et Pusch 2007 ; Zając et Zając, 2011), ou pourraient aussi être la conséquence du comportement reproducteur particulier d'U. crassus (Vicentini, 2005). Par ailleurs, le caractère défavorable des profondeurs importantes est peut-être dû au fait qu'une augmentation de la hauteur d'eau provoque une élévation des contraintes de cisaillement (Leopold et al., 1964). Dans la même logique, les mulettes épaisses pourraient éviter les mouilles car les contraintes de cisaillement y sont plus importantes en cas de fort débit (Petit, 1987 ; Zając et Zając, 2011). Cette contrainte exercée par le flux semble en effet négativement corrélée avec la densité d'U. crassus (Stoeckl et Geist, 2016).

\subsection{Substrats occupés par U. crassus}

Une large diversité de faciès sédimentaires sont présents dans le lit mineur de la Sûre, et les mulettes colonisent l'ensemble des types de dépôts, depuis ceux issus de la suspension uniforme jusqu'à ceux résultant du roulage. La granularité des éléments constituant le substrat peut influencer les populations d'U. crassus, surtout en regard des éléments grossiers (Zając et Zając, 2011 ; Klos et al., 2015). Le substrat principalement colonisé par U. crassus dans la Sûre présente un $D_{50}$ de l'ordre de $3 \mathrm{~mm}$, un $D_{90}$ de l'ordre de $11 \mathrm{~mm}$, et un $D_{99}$ de l'ordre de $26 \mathrm{~mm}$ ce qui correspond à du gravier sableux argilo-limoneux ou du gravier sableux. En Suède, Pettersson (2012) propose un substrat optimal avec un $D_{50}$ de $4 \mathrm{~mm}$ (gravier fin), ce qui est confirmé sur le terrain par l'équipe du projet LIFE (UC4Life). Lors des recensements réalisés dans le cadre de ce projet, des populations d'U. crassus ont aussi été observées dans des substrats plus grossiers présentant un $D_{50}$ de $60 \mathrm{~mm}$ (Lundberg et Österling, 2016). En outre, une synthèse réalisée en Allemagne indique que les mulettes épaisses adultes se trouvent plus volontiers dans le sable grossier $(0,85 \mathrm{~mm}$ à $2 \mathrm{~mm})$ et le gravier fin $(2 \mathrm{~mm}$ à $6,3 \mathrm{~mm})$ mais aussi dans des substrats plus fins $(<0,85 \mathrm{~mm}$ ) (Hochwald, 2012). L'ensemble de ces valeurs concordent en indiquant qu'U. crassus vit préférentiellement dans un substrat relativement peu grossier, et présentant un pourcentage important de matières fines $(<2000 \mu \mathrm{m})$. Ce type de substrat, meuble, permet probablement de s'y enfoncer facilement et rapidement pour échapper à un danger (crue, prédateur, compétiteur) (Schwalb et Pusch, 2007). L'analyse des échantillons de substrat révèle également que l'espèce peut se développer dans des sédiments fins $\left(D_{50}\right.$ de $\left.30 \mu \mathrm{m}\right)$. Ces résultats sont en accord avec ceux apportés par Zajac et al. (2018), et Denic et al. (2014) admettant que les mulettes épaisses peuvent se développer dans des zones d'accumulation de sédiments fins à faible résistance de pénétration (Stoeckl et Geist, 2016). Cependant, dans la Sûre, il semble que des dépôts avec un faible pourcentage de matières organiques soient privilégiés, potentiellement afin d'éviter l'anoxie.

\subsection{Colmatage et mobilité des sédiments}

Les pièges à sédiments collectent la fraction $<5600 \mu \mathrm{m}$ de la charge de fond en mouvement, qui après avoir été mobilisée par le courant, se dépose à la surface du lit ou s'y infiltre. Les mouvements de sédiments dus aux courants interstitiels sont bloqués par la configuration du piège (Casas-Mulet et al., 2017). Le taux de remplissage des pièges s'est avéré relativement faible durant la période d'étude, variant entre 4 et $14 \%$ pour des débits compris entre 0,1 et $0,3 Q_{b}$. La fraction $<2000 \mu \mathrm{m}$ représentait 3 à $11 \%$ du remplissage suivant les cas. Les variations de remplissage des pièges ne semblent pas pouvoir être expliquées par les faibles différences entre les pentes locales des sites ( $\pm 0,5 \%$ ), ni par les différences de débits. À titre de comparaison, un taux de remplissage de 2 à $4 \%$ par mois par la fraction fine $(<2000 \mu \mathrm{m})$ a été observé lors d'un suivi sédimentaire dans un secteur plus en amont de la Sûre pour un débit maximal similaire (BV: $40 \mathrm{~km}^{2}$, débit max : 0,26 $Q_{b}$, pente locale : $6 \%$ ) (Vaessen et al., 2019). Il est probable que la pente du plan d'eau plus importante de ce secteur $(+4 \%$ ) explique le plus faible taux de sédimentation mesuré. La variabilité saisonnière du transport 
sédimentaire pourrait également expliquer les différences observées dans les taux de remplissage. En effet, les périodes de cultures et le pâturage peuvent influencer le taux de matières en suspension (Steegen et al., 1998), de même que l'érosion des berges et du lit en relation notamment avec les alternances de gel et de dégel (Wallemacq et al., 2011). Cependant, le taux de matières en suspension dans la Sûre reste faible de manière générale $\left(<40 \mathrm{mg} / \mathrm{l}\right.$ au $\left.Q_{b}\right)$. Finalement, il est à noter que les taux d'infiltration de sédiments fins dans les pièges ne sont pas en adéquation avec les niveaux de colmatage mis en évidence par les sticks hypoxie. Le colmatage, lorsqu'il se produit sur la Sûre, ce qui semble rare, pourrait résulter d'un processus plus long, voire exceptionnel, non mis en évidence lors de la période de suivi sédimentaire de cette étude.

Le transport de la charge de fond se produit via différents processus liés à la vitesse du courant et la taille des particules (Bravard et Petit, 2000). Le transport de la charge de fond dans les rivières ardennaises ayant un bassin versant entre 100 et $500 \mathrm{~km}^{2}$ se produit généralement pour un débit avoisinant les $0,5 Q_{b}$ (Houbrechts et al., 2006). Les débits $\geq 0,5 Q_{b}$ se produisent en moyenne 11 jours par an dans la Surre. Le débit de mise en mouvement de la charge de fond de la Sûre n'a pu être identifié précisément grâce aux relevés des galets marqués. En effet, les données des PIT Tags, couplées à celles des chaînes d'érosion, permettent seulement de situer le débit mobilisateur entre 0,3 et $1,3 Q_{b}$. Grâce à la relation établie par Houbrechts et al. (2015) entre la taille des dix plus gros éléments mobilisés et la puissance spécifique, il semble que le débit mobilisateur soit d'une puissance spécifique l'ordre de $33 \mathrm{~W} / \mathrm{m}^{2}$. Cette puissance spécifique correspond à un débit qui se produit en moyenne 4 jours par an, ce qui implique une fréquence de charriage relativement faible. Par ailleurs, le suivi des galets marqués a permis de mettre en évidence que pour une crue légèrement supérieure au Qb $\left(1,3 \mathrm{Qb}, 51 \mathrm{~W} / \mathrm{m}^{2}\right.$, site 2), la Sûre mobilise des éléments de l'ordre de $36 \mathrm{~mm}$ d'axe $\mathrm{b}$ en moyenne. Cette crue de $51 \mathrm{~W} / \mathrm{m}^{2}$ a déplacé des galets pouvant aller jusqu'à 55 $\mathrm{mm}$ d'axe b. La plupart des mulettes épaisses recensées présentant une taille $<55 \mathrm{~mm}$, elles peuvent être facilement emportées si elles ne sont pas ancrées dans le substrat. En effet, si elles sont enfouies dans le substrat, et que le substrat est suffisamment stable, les adultes peuvent alors résister à de telles crues (May et Pryor, 2016). Ainsi, ces organismes sont vulnérables aux événements hydrologiques extrêmes qui peuvent briser l'armurage et remanier la sous-couche $\mathrm{du}$ lit. Lors de tels événements, la rivière peut alors emporter les mulettes, et par là même, entrainer une mortalité importante dans les populations. Par exemple, en 2003, suite à une crue de $142 \mathrm{~W} / \mathrm{m}^{2}$ dans la Sûre $\left(88,4 \mathrm{~m}^{3} / \mathrm{s}\right.$, temps de retour de 30 ans), au moins 150 individus $U$. crassus récemment morts ont été trouvés déposés sur la plaine alluviale, de même qu'un grand nombre de coquilles vides (Laudelout et Libois, 2003). En Écosse en 1998, une crue de récurrence égale à 100 ans a mené à une perte de 4 à $8 \%$ de la population totale de moules perlières, un bivalve de la même famille qu'U. crassus (Hastie et al., 2001). Une mulette épaisse peut vivre jusqu'à 30 ans ce qui la rend susceptible d'être confrontée à des événements hydrologiques extrêmes, mais ce qui entraine surtout qu'elle devra faire face au cours de sa vie à de nombreux événements morphogènes.

Hastie et al. (2001) notent, suite à la crue centennale, une mortalité des bivalves plus faible dans les secteurs avec un lit composé de gros éléments. L'hétérogénéité du substrat fournirait des zones refuges pour les mulettes les protégeant du stress pouvant être induit par les conditions de forts débits (Strayer, 1999). Cependant, bien que le lit de la Sûre dans certains secteurs présente des blocs, ce n'est pas le cas à proximité immédiate des mulettes épaisses $\left(D_{99}\right.$ maximal était de $90 \mathrm{~mm}$ ). Houbrechts et al. (2012) ont mesuré une épaisseur de couche active de $15 \mathrm{~mm}$ dans la Sûre en tête de bassin (BV : $31 \mathrm{~km}^{2}$ ) suite à une crue de puissance spécifique $16 \mathrm{~W} / \mathrm{m}^{2}$ pour un débit de 0,5 Qb. Par ailleurs, les auteurs mettent en évidence que l'épaisseur de couche de charriage est systématiquement inférieure au $\mathrm{D}_{50}$ des seuils (Houbrechts et al., 2012), qui pour rappel est de $3 \mathrm{~cm}$ au niveau des sites d'étude. Ainsi, il est probable que les mulettes épaisses de la Sûre résistent aux débits mobilisateurs en s'enfouissant rapidement dans le substrat plus profondément que l'épaisseur de couche active de remaniement.

Ensemble, la granularité du substrat, le faible colmatage du lit des zones colonisées, l'épaisseur peu importante de la couche active mobilisée lors du débit pleins bords, et de manière générale la mobilisation relativement peu importante entrainée par une crue proche du $Q_{b}$ semblent indiquer qu'il existe dans la Sûre un compromis adéquat pour les mulettes épaisses, fournissant des zones meubles et oxygénées, et suffisamment stables pour résister aux crues.

\section{Conclusion}

L'étude de la répartition spatiale d'U. crassus dans différents microhabitats d'une rivière à charge caillouteuse a permis de mettre en évidence l'importance prépondérante de la hauteur d'eau pour des débits inférieurs au module. Pour ces débits, l'espèce vit principalement dans des zones de 0,4 à $0,8 \mathrm{~m}$ de profondeur, et présentant une vitesse de courant faible mais non nulle $(<0,4 \mathrm{~m} / \mathrm{s})$. Les mulettes occupent des substrats variés mais la plupart se trouvent dans des graviers fins sableux $\left(D_{50}=3 \mathrm{~mm}, D_{90}=11 \mathrm{~mm}\right.$, $D_{99}=26 \mathrm{~mm}$ ). Les échantillons de substrat sont constitués d'une part importante de sédiments peu grossiers $(<2000 \mu \mathrm{m})$ et ont une faible teneur en matières organiques. La fréquence de charriage limitée pourrait être une caractéristique appréciée par l'espèce. La définition des microhabitats optimaux d'U. crassus peut être complexe car ceux-ci sont attribuables à des caractéristiques liées aux conditions hydrologiques (substrat bien trié, accumulation de sédiments fins, stabilité, etc.). Malgré tout, l'occupation des microhabitats dans la Sûre indique la capacité d'U. crassus à coloniser une gamme étendue de conditions hydromorphologiques, tout en ayant des préférences marquées. L'étude de l'habitat, et des relations que l'espèce entretient avec lui, est une étape primordiale dans l'amélioration de sa protection. Or, la sauvegarde des espèces endémiques de bivalves filtreurs relève de l'urgence au regard de la dégradation actuelle des écosystèmes d'eau douce. La définition des intervalles de tolérance concernant les variables définissant l'habitat d'U. crassus permettra de faire progresser sa conservation en Europe dans le cadre de la Directive 92/43/CEE.

\section{Remerciements}

Les auteurs remercient Nicolas Mayon du Parc naturel HauteSûre Forêt d'Anlier, et Grégory Motte du Département de l'Étude du milieu naturel et agricole du Service public de Wallonie pour leur participation active à cette étude. Alain Hambuckers (ULiège) est également chaleureusement remercié pour ses conseils. Nous 
remercions finalement deux relecteurs anonymes pour leurs commentaires pertinents. Cet article a été rédigé avec le soutien de la Communauté française de Belgique dans le cadre du financement d'une bourse FRIA, ainsi qu'avec le soutien de l'UR Sphère.

\section{Références}

Ackerman J. D. (1999) - Effect of velocity on the filter feeding of dreissenid mussels (Dreissena polymorpha and Dreissena bugensis) : Implications for trophic dynamics. Canadian Journal of Fisheries and Aquatic Sciences, 56 (9), 1551-1561.

DOI : $10.1139 / \mathrm{f} 99-079$

Aldridge D. C., Fayle T. M., Jackson N. (2007) - Freshwater mussel abundance predicts biodiversity in UK lowland rivers. Aquatic Conservation : Marine and Freshwater Ecosystems, 17 (6), 554564.

\section{DOI : $10.1002 /$ aqc. 815}

Blott S. J., Pye K. (2001) - GRADISTAT : A grain size distribution and statistics package for the analysis of unconsolidated sediments. Earth Surface Processes and Landforms, 26 (11), $1237-1248$.

DOI : $10.1002 /$ esp. 261

Bogan A. E. (1993) - Freshwater bivalve extinctions (Mollusca : Unionoida) : a search for causes. American Zoologist, 33 (6), 599609.

DOI : $10.1093 / \mathrm{icb} / 33.6 .599$

Bovee K. D. (1986) - Development and evaluation of habitat suitability criteria for use in the Instream Flow Incremental Methodology. Instream Flow Information Paper 21. U.S. Fish and Wildlife Service, Biological Report, 86 (7), 235 p.

Box J. B., Dorazio R. M., Liddell W. D. (2002) - Relationships between streambed substrate characteristics and freshwater mussels (Bivalvia : Unionidae) in Coastal Plain streams. Journal of the North American Benthological Society, 21 (2), 253-260. DOI : $10.2307 / 1468413$

Bravard J.-P., Petit F. (2000) - Les cours d'eau : Dynamique du système fluvial. Armand Colin, Paris, 222 p.

Bunte K., Abt S. R. (2001) - Sampling surface and subsurface particle-size distributions in wadable gravel- and cobble-bed streams for analyses in sediment transport, hydraulics, and streambed monitoring. US Department of Agriculture, Forest Service, Rocky Mountain Research Station, 428 p. DOI : $10.2737 /$ RMRS-GTR-74

Casas-Mulet R., Alfredsen K. T., McCluskey A. H., Stewardson M. J. (2017) - Key hydraulic drivers and patterns of fine sediment accumulation in gravel streambeds : A conceptual framework illustrated with a case study from the Kiewa River, Australia. Geomorphology, 299, 152-164.

DOI : 10.1016/j.geomorph.2017.08.032

Castelain L., Peeters A., Halleux M., de le Court B., Verniers G. (2018) - Walphy, un projet expérimental de réhabilitation de cours d'eau : suivis hydromorphologiques et écologiques. Hydroécologie Appliquée, 20, 29-55.

Dascher E. D., Burlakova L. E., Karatayev A. Y., Ford D. F., Schwalb A. N. (2018) - Distribution of unionid freshwater mussels and host fishes in Texas. A study of broad-scale spatial patterns across basins and a strong climate gradient. Hydrobiologia, 810, 315-331.
DOI : $10.1007 / \mathrm{s} 10750-017-3168-5$

Datry T., Dole-Olivier M. J., Marmonier P., Claret C., Perrin J. F., Lafont M., Breil P. (2008) - La zone hyporhéique, une composante à ne pas négliger dans l'état des lieux et la restauration des cours d'eau. Ingénieries eau-agriculture-territoires, 54, 3-18.

Denic M., Stoeckl K., Gum B., Geist J. (2014) - Physicochemical assessment of Unio crassus habitat quality in a small upland stream and implications for conservation. Hydrobiologia, 735 (1), 111-122.

DOI : $10.1007 / \mathrm{s} 10750-013-1467-\mathrm{Z}$

Díaz S., Settele J., Brondízio E., Ngo H. T., Guèze M., Agard J., Arneth A., Balvanera P., Brauman K., Butchart S., Chan K., Garibaldi L., Ichii K., Liu J., Subramanian S. M., Midgley G., Miloslavic P., Molnár Z., Obura D., Pfaff A., Polasky P., Purvis A., Razzaque J., Reyers B., Chowdhury R. R., Shin Y.-J., Visseren-Hamakers I., Willis K., Zayas C. (2019) - Summary for policymakers of the global assessment report on biodiversity and ecosystem services of the Intergovernmental SciencePolicy Platform on Biodiversity and Ecosystem Services. IPBES secretariat, Bonn, $56 \mathrm{p}$.

Dobler A. H., Geist J., Stoeckl K., Inoue K. (2019) - A spatially explicit approach to prioritize protection areas for endangered freshwater mussels. Aquatic Conservation : Marine and Freshwater Ecosystems, 29 (1), 12-23.

DOI : $10.1002 /$ aqc. 2993

Douda K. (2007) - The occurrence and growth of Unio crassus (mollusca : Bivalvia : Unionidae) in Lužnice river basin in respect to water quality. Acta Universitatis Carolinae, Environmentalica, 21, 57-63.

Downing J. A., Van Meter P., Woolnough D. A. (2010) - Suspects and evidence : a review of the causes of extirpation and decline in freshwater mussels. Animal Biodiversity and Conservation, 33 (2), 151-185.

Duerdoth C. P., Arnold A., Murphy J. F., Naden P. S., Scarlett P., Collins A. L., Sear D. A., Jones, J. I. (2015) - Assessment of a rapid method for quantitative reach-scale estimates of deposited fine sediment in rivers. Geomorphology, 230, 37-50.

DOI : $10.1016 /$ j.geomorph.2014.11.003

Dunca E. (2014) - Age determination of Unio crassus shells from Sauer and Our Rivers. Bivalvia Rapport 9, 20 p. (non publié).

Engel H., Wächtler K. (1989) - Some peculiarities in developmental biology of two forms of the freshwater bivalve Unio crassus in northern Germany. Archiv für Hydrobiologie, 115 (3), 441-450.

Engler R., Guisan A., Rechsteiner L. (2004) - An improved approach for predicting the distribution of rare and endangered species from occurrence and pseudo-absence data. Journal of Applied Ecology, 41 (2), 263-274.

DOI : 10.1111/j.0021-8901.2004.00881.x

Ferreira-Rodríguez N., Akiyama Y. B., Aksenova O. V., Araujo R., Barnhart M. C., Bespalaya Y. V., Bogan A. E., Bolotov I. N., Budha P. B., Clavijo C., Clearwater S. J., Darrigran G., Do V. T., Douda K., Froufe E., Gumpinger C., Henrikson L., Humphrey C. L., Johnson N. A., Klishko O., Klunzinge M. W., Kovitvadhi S., Kovitvadhi U., Lajtner J., Lopes-Lima M., Moorkens E. A., Nagayama S., Nagel K.-O., Nakano M., Negishi J. N., Ondina P., Oulasvirta P., Prié V., Riccardi N., Rudzīte M., Sheldon F., Sousa R., Strayer D. L., Takeuchi M., Taskinen J., Teixeira 
A., Tiemann J. S., Urbańska M., Varandas S., Vinarski M. V., Wicklow B. J., Zając T., Vaughn C. C. (2019) - Research priorities for freshwater mussel conservation assessment. Biological Conservation, 231, 77-87.

DOI : 10.1016/j.biocon.2019.01.002

Folk R. L. (1954) - The distinction between grain size and mineral composition in sedimentary-rock nomenclature. The Journal of Geology, 62 (4), 344-359.

DOI : $10.1086 / 626171$

Fouillé S. (2013) - Étude d'impact de la qualité intra-sédimentaire en vue de la protection des naïades sur les rivières de la Cure et du Cousin. Mémoire de Master, Université de Rennes 1, 76 p.

Frostick L. E., Lucas P. M., Reid I. (1984) - The infiltration of fine matrices into coarse-grained alluvial sediments and its implications for stratigraphical interpretation. Journal of the Geological Society, 141 (6), 955-965.

DOI : 10.1144 /gsigs.141.6.0955

Gates K. K., Vaughn C. C., Julian J. P. (2015) - Developing environmental flow recommendations for freshwater mussels using the biological traits of species guilds. Freshwater Biology, 60 (4), 620-635.

DOI : $10.1111 /$ fwb. 12528

Geist J., Hawkins S. J. (2016) - Habitat recovery and restoration in aquatic ecosystems : current progress and future challenges. Aquatic Conservation : Marine and Freshwater Ecosystems, 26 (5), 942-962.

DOI : $10.1002 /$ aqc. 2702

Gutiérrez J. L., Jones C. G., Strayer D. L., Iribarne O. O. (2003) Mollusks as ecosystem engineers : The role of shell production in aquatic habitats. Oikos, 101 (1), 79-90.

DOI : $10.1034 /$ j.1600-0706.2003.12322.x

Hastie L. C., Boon P. J., Young M. R. (2000) - Physical microhabitat requirements of freshwater pearl mussels, Margaritifera margaritifera (L.). Hydrobiologia, 429, 59-71.

DOI : $10.1023 / \mathrm{A}: 1004068412666$

Hastie L. C., Boon P. J., Young M. R., Way S. (2001) - The effects of a major flood on an endangered freshwater mussel population. Biological Conservation, 98 (1), 107-115.

DOI : $10.1016 / \mathrm{S} 0006-3207(00) 00152-\mathrm{X}$

Hochwald S. (2001) - Plasticity of life-history traits in Unio crassus. In Bauer G., Wächtler K. (Eds) : Ecology and evolution of the freshwater mussels Unionoida. Springer-Verlag Berlin Heidelberg, 127-141.

Hochwald S., Gum B., Rudolph B.-U., Sachteleben J. (2012) - Leitfaden Bachmuschelschutz. Bayerisches Landesamt für Umwelt (LfU), Augsburg, 116 p. (non publié).

Houbrechts G., Hallot É., Gob F., Mols J., Defechereux O., Petit F. (2006) - Fréquence et importance du charriage dans les rivières du Massif ardennais. Géographie physique et Quaternaire, 60 (3), 241-252.

\section{DOI : $10.7202 / 017998 \mathrm{ar}$}

Houbrechts G., Van Campenhout J., Levecq Y., Hallot E., Peeters A., Petit F. (2012) - Comparison of methods for quantifying active layer dynamics and bedload discharge in armoured gravelbed rivers. Earth Surface Processes and Landforms, 37 (14), 15011517.

DOI : $10.1002 /$ esp. 3258
Houbrechts G., Hallot E., Levecq Y., Denis A. C., Van Campenhout J., Peeters A., Petit F. (2013) - Images CM de Passega des rivières ardennaises. Bulletin de la Société Géographique de Liège, 61, 37-68.

Houbrechts G., Levecq Y., Peeters A., Hallot E., Van Campenhout J., Denis A. C., Petit F. (2015) - Evaluation of long-term bedload virtual velocity in gravel-bed rivers (Ardenne, Belgium). Geomorphology, 251, 6-19.

DOI : 10.1016/j.geomorph.2015.05.012

Inoue K., Stoeckl K., Geist J. (2017) - Joint species models reveal the effects of environment on community assemblage of freshwater mussels and fishes in European rivers. Diversity and Distributions, 23 (3), 284-296.

DOI : $10.1111 /$ ddi. 12520

Jansen W., Bauer G., Zahner-Meike E. (2001) - Glochidial mortality in freshwater mussels. In Bauer G., Wächtler K. (eds) : Ecology and evolution of the freshwater mussels Unionoida. Springer-Verlag Berlin Heidelberg, 185-211.

Klos P. Z., Rosenberry D. O., Nelson G. R. (2015) - Influence of hyporheic exchange, substrate distribution, and other physically linked hydrogeomorphic characteristics on abundance of freshwater mussels. Ecohydrology, 8 (7), 1284-1291.

DOI : $10.1002 /$ eco.1581

Knighton D. (1998) - Fluvial forms and processes : a new perspective. Arnold, London, $383 \mathrm{p}$.

Lamand F., Beisel J.-N. (2014) - Comparison of visual observation and excavation to quantify density of the endangered bivalve Unio crassus in rivers of north-eastern France. Knowledge and Management of Aquatic Ecosystems, 413, 11. DOI : $10.1051 / \mathrm{kmae} / 2014009$

Lamouroux N., Hauer C., Stewardson M. J., Poff N. L. (2017) Physical habitat modeling and ecohydrological tools. In Horne A. C., Webb J. A., Stewardson M. J., Richter B., Acreman M. (eds) : Water for the environment. Academic Press. 265-285. DOI : $10.1016 /$ B978-0-12-803907-6.00013-9

Laronne J. B., Outhet D. N., Carling P. A., McCabe T. J. (1994) Scour Chain Employment in Gravel-Bed Rivers. Catena, 22 (4), 299-306.

DOI : $10.1016 / 0341-8162(94) 90040-\mathrm{x}$

Laudelout A., Libois R. (2003) - Rapport sur les espèces macroindicatrices de la qualité des cours d'eau. Répartition, Habitat, Mesures de protection et de restauration des populations. Ministère de la Région wallonne : Direction générale des Ressources naturelles et de l'Environnement, Division de l'Eau, Jambes, $142 \mathrm{p}$.

Layzer J. B., Madison L. M. (1995) - Microhabitat use by freshwater mussels and recommendations for determining their instream flow needs. Regulated Rivers : Research \& Management, 10 (2-4), 329-345.

DOI : $10.1002 /$ rrr.3450100225

Leopold L., Wolman M., Miller J. (1964) - Fluvial Processes in Geomorphology. Freeman and Co, San Francisco, 522 p.

Lopes-Lima M., Kebapçı U., Van Damme D. (2014) - Unio crassus. The IUCN Red List of Threatened Species 2014 : e.T22736A42465628.

DOI : 10.2305/IUCN.UK.2014- 1.RLTS.T22736A42465628.en

Lopes-Lima M., Burlakova L. E., Karatayev A. Y., Mehler K., 
Seddon M., Sousa R. (2018) - Conservation of freshwater bivalves at the global scale : diversity, threats and research needs. Hydrobiologia, 810 (1), 1-14.

DOI : $10.1007 / \mathrm{s} 10750-017-3486-7$

Lundberg S., Österling M. (2016) - Return of the Thick-shelled River Mussel - Restoring floodplains, habitats and connectivity by using mussels and brains. Handbook, UC4LIFE project. Skåne County Administrative Board, 99 p. (non publié).

Marmonier P., Delettre Y., Lefebvre S., Guyon J., Boulton A. J. (2004) - A simple technique using wooden stakes to estimate vertical patterns of interstitial oxygenation in the beds of rivers. Archiv für Hydrobiologie, 160 (1), 133-143. DOI : $10.1127 / 0003-9136 / 2004 / 0160-0133$

May C. L., Pryor B. S. (2016) - Explaining spatial patterns of mussel beds in a Northern California river : The role of flood disturbance and spawning salmon. River Research and Applications, 32 (4), 776-785. DOI : $10.1002 /$ rra.2894

Morales Y., Weber L. J., Mynett A. E., Newton T. J. (2006) - Effect of substrate and hydrodynamic conditions on the formation of mussel beds in a large river. Journal of the North American Benthological Society, 25 (3), 664-676. DOI : 10.1899/0887-3593(2006)25[664 :EOSAHC]2.0.CO ;2

Passega R. (1957) - Texture as characteristic of clastic deposition. AAPG Bulletin, 41 (9), 1952-1984.

DOI : 10.1306/0BDA594E-16BD-11D7-8645000102C1865D

Passega R. (1964) - Grain size representation by CM patterns as a geologic tool. Journal of Sedimentary Research, 34, 830-847. DOI : $10.1306 / 74 D 711$ A4-2B21-11D7-8648000102C1865D

Peeters A., Houbrechts G., Hallot E., Campenhout J. V., Verniers G., Petit F. (2018) - Efficacité et résistance de techniques de protection de berges en génie végétal. Géomorphologie : Relief, Processus, Environnement, 24 (2), 121-138.

DOI : 10.4000 /geomorphologie.11927

Petit F. (1987) - The relationship between shear stress and the shaping of the bed of a pebble-loaded river La Rulles - Ardenne. Catena. 14 (5), 453-468.

DOI : $10.1016 / 0341-8162(87) 90015-4$

Pettersson E. (2012) - Vilken preferens har tjockskalig målarmussla (Unio crassus) för bottensubstrat och vattenhastighet i Storån, Östergötland ? Thèse de bachelier, Université de Linköping, 26 p.

Piégay H., Arnaud F., Cassel M., Dépret T., Alber A., Michel K., Rollet A.-J., Vaudor L. (2016) - Suivi par RFID de la mobilité des galets : retour sur 10 ans d'expérience en grandes rivières. Bulletin de la Société Géographique de Liège, 67, 77-91.

Schmitz C., Krippel Y. (2007) - Mise en place d'un plan de gestion transfrontalier des ressources en eau de la Haute-Sûre belgoluxembourgeoise. Contrat de Rivière Haute-Sûre, 135 p. (non publié).

Schneider L. D., Nilsson P. A., Österling E. M. (2018) - Evaluating temperature-and host-dependent reproduction in the parasitic freshwater mussel Unio crassus. Hydrobiologia, 810, 283-293. DOI : $10.1007 /$ s10750-017-3217-0

Schwalb A. N., Pusch M. T. (2007) - Horizontal and vertical movements of unionid mussels in a lowland river. Journal of the North American Benthological Society, 26 (2), 261-272. DOI : 10.1899/0887-3593(2007)26[261 :HAVMOU]2.0.CO ;2
Schwalb A. N., Morris T. J., Mandrak N. E., Cottenie K. (2013) - Distribution of unionid freshwater mussels depends on the distribution of host fishes on a regional scale. Diversity and Distributions, 19 (4), 446-454.

DOI : 10.1111/j.1472-4642.2012.00940.x

Souchon Y., Trocherie F., Fragnoud E., Lacombe C. (1989) - Les modèles numériques des microhabitats des passons : Application et nouveaux développements. Revue des sciences de l'eau, 2 (4), 807-830.

DOI : $10.7202 / 705056 \mathrm{ar}$

Steegen A., Govers G., Beuselinck L., Nachtergaele J., Takken I., Poesen J. (1998) - Variations in sediment yield from an agricultural drainage basin in central Belgium. In Summer W., Klaghofer E., Zhang W. (eds) : Modelling soil erosion, sediment transport and closely related hydrological processes. IAHS Publication no. 249, 177-185.

Steuer J. J., Newton T. J., Zigler S. J. (2008) - Use of complex hydraulic variables to predict the distribution and density of unionids in a side channel of the Upper Mississippi River. Hydrobiologia, 610 (1), 67-82.

DOI : $10.1007 / \mathrm{s} 10750-008-9423-\mathrm{Z}$

Stoeckl K., Geist J. (2016) - Hydrological and substrate requirements of the thick-shelled river mussel Unio crassus (Philipsson 1788). Aquatic Conservation : Marine and Freshwater Ecosystems, 26 (3), 456-469.

DOI : $10.1002 /$ aqc. 2598

Strayer D. L. (1999) - Use of flow refuges by unionid mussels in rivers. Journal of the North American Benthological Society, 18 (4), 468-476. DOI : $10.2307 / 1468379$

Taeubert J. E., Gum B., Geist J. (2012) - Host-specificity of the endangered thick-shelled river mussel (Unio crassus, Philipsson 1788) and implications for conservation. Aquatic Conservation : Marine and Freshwater Ecosystems, 22 (1), 36-46.

DOI : $10.1002 /$ aqc. 1245

Vaessen Q., Peeters A., Van Campenhout J., Houbrechts G. (2019) - Suivi de l'impact hydromorphologique du démontage de barrages de castor sur la Sûre (phase 1). Rapport du Laboratoire d'hydrographie et de géomorphologie fluviatile (ULiège), $27 \mathrm{p}$. (non publié).

Van Campenhout J., Denis A. C., Hallot É., Houbrechts G., Levecq Y., Peeters A., Petit F. (2013) - Flux des sédiments en suspension dans les rivières du bassin de la Meuse : proposition d'une typologie régionale basée sur la dénudation spécifique des bassins versants. Bulletin de la Société géographique de Liège, 61, 15-36.

Van Campenhout J., Houbrechts G., Peeters A., Petit F. (2020) Return period of characteristic discharges from the comparison between partial duration and annual series, application to the Walloon Rivers (Belgium). Water, 12 (3), 792.

DOI : $10.3390 /$ w12030792

Vaughn C. C. (2017) - Ecosystem services provided by freshwater mussels. Hydrobiologia, 810 (1), 15-27.

DOI : $10.1007 / \mathrm{s} 10750-017-3139-\mathrm{x}$

Vicentini H. (2005) - Unusual spurting behaviour of the freshwater mussel Unio crassus. Journal of Molluscan Studies, 71 (4), 409410. 
DOI : 10.1093/mollus/eyi045

Wallemacq V., Petit F., Van Campenhout J., Houbrechts G. (2011) - Impact de la glace de ségrégation sur l'érosion des berges d'un ruisseau ardennais (la Chavanne, Belgique). Géomorphologie : Relief, Processus, Environnement, 17 (1), 65-82.

DOI : $10.4000 /$ geomorphologie.9226

Wolman G. (1954) - A method of sampling coarse river-bed material. EOS, Transactions American Geophysical Union, 35 (6), 951-956.

DOI : $10.1029 /$ TR035i006p00951

Wood P. J., Armitage P. D. (1999) - Sediment deposition in a small lowland stream-management implications. Regulated Rivers : Research \& Management, 15 (1-3), 199-210.

DOI : 10.1002/(SICI)1099-1646(199901/06)15:1/3<199 : :AID$\underline{\mathrm{RRR} 531>3.0 . \mathrm{CO} ; 2-0}$

Zając K., Zając T. (2011) - The role of active individual movement in habitat selection in the endangered freshwater mussel Unio crassus Philipsson 1788. Journal of Conchology, 40 (4), 446-461.

Zając K., Florek J., Zając T., Adamski P., Bielański W., Ćmiel A. M., Klich M., Lipińska A. M. (2018) - On the reintroduction of the endangered thick-shelled river mussel Unio crassus : The importance of the river's longitudinal profile. Science of the Total Environment, 624, 273-282.

DOI : $10.1016 /$ j.scitotenv.2017.11.346

Zettler M. L., Jueg U. (2007) - The situation of the freshwater mussel Unio crassus (Philipsson, 1788) in north-east Germany and its monitoring in terms of the EC Habitats Directive. Mollusca, 25 (2), 165-174.

Zieritz A., Geist J., Gum, B. (2014) - Spatio-temporal distribution patterns of three stream-dwelling freshwater mussel species : towards a strategy for representative surveys. Hydrobiologia, 735 (1), 123-136.

DOI : $10.1007 / \mathrm{s} 10750-014-1897-2$

\section{Abridged english version}

Once widely distributed in European rivers, the thick shelled river mussel (Unio crassus), a freshwater mussel of the family Unionidae, is now at a high risk of extinction. This species and its habitat are protected in Europe by Council Directive 92/43/EEC (Annexes II and $I V)$ on the conservation of natural habitats and of wild fauna and flora. Member States have to establish conservation strategies for this species. However, many uncertainties about its habitat remain. The objective of this study is to define the characteristics of the microhabitats of U. crassus in a Belgian river (the Sûre River) with recruiting populations by: (i) analysing the spatial distribution of individuals at several sections of the river, (ii) determining the relative importance of the measured microhabitat variables in the presencel absence of the species, and (iii) examining sediment transport and its possible impact on the species.

The Surre River is a gravel-bed river that originates in the SouthEast of Belgium, in the Ardennes. It has a width lower than $15 \mathrm{~m}$ $\left(w_{Q b}\right)$, an average slope of $2.2 \%$ and a bankfull unit stream power $\left(\omega_{Q b}\right)$ of approximately $52 \mathrm{~W} / \mathrm{m}^{2}$. To identify the hydromorphological preferences of $U$. crassus, bathymetry and distribution of flow velocities were mapped at three study sites using a microhabitat approach. Moreover, individuals were surveyed at these study sites in order to map their spatial distribution according to the different classes of microhabitats (depth and velocity). Habitat suitability indexes were calculated by relating the use of different microhabitats by the individuals to the availability of these same microhabitats. The relative effect of depth, current velocity and distance from the bank on the presencelabsence of the species was analysed with a logistic model. On the other hand, the characteristic grain size indices of the substrate were determined by taking samples where the mussels were located. The clogging of substrate in the study sites was assessed using sediment traps. These devices trap sediments finer than 5,600 $\mu \mathrm{m}$ by mimicking the natural gravel layer, which makes it possible to quantify the infiltration rate of the fine fraction. In addition, pebbles marked with PIT Tags have been injected in order to monitor the dynamics of coarse bedload in relation to hydrological events.

At study sites, and for a discharge below the mean annual flow, the thick shelled river mussels were mainly observed in lateral deposits or in point bar deposits. Most individuals were located in areas with an intermediate depth; between 0.4 and $0.8 \mathrm{~m}$ (74\% of the individuals). The habitat suitability index suggests that shallow depths $(<0.2 \mathrm{~m})$ are also suitable for the species. Flow velocities below $0.4 \mathrm{~m} / \mathrm{s}$ appear to be particularly adequate, with $87 \%$ of the individuals recorded. The habitat suitability index indicates that areas with faster flows may not be prohibitive. Depth appears to be the main factor affecting this spatial distribution. The substrate inhabited by U. crassus has a median $D_{50}$ of $3 \mathrm{~mm}$ and a median $D_{99}$ of $26 \mathrm{~mm}$. The majority of individuals (72\%) have been found in muddy sandy gravel or in sandy gravel (Folk and Ward classification). The species can also be found in much finer substrates $\left(D_{50}=30 \mu \mathrm{m}\right)$. Infiltration rates of fine sediments on the Surre River are low (3 to $11 \%$ of the spaces filled by the $<2000 \mu \mathrm{m}$ fraction) although some variability is observed between the study periods. This variability is not explained by the occurrence of flood events or by the differences in the local geomorphological settings of the study sites such as the local slope, but probably by seasonal variations (farming, frost season). Monitoring of marked pebbles with PIT Tags showed that a $51 \mathrm{~W} / \mathrm{m}^{2}$ flood could mobilize pebbles of $36 \mathrm{~mm}$ in average, and up to $55 \mathrm{~mm}$, over a mean distance of $24 \mathrm{~m}$. Such low-magnitude flood (Return Interval $\sim 1 \mathrm{yr}$ ) does not seem to present any risk for thick shelled river mussels which are able to burrow into the substrate. By contributing to the definition of tolerance intervals and by improving the knowledge of the relations that the thick shelled river mussel maintains with its habitat, this study is a step in the improvement of its conservation in Europe. 